\title{
MEIOSIS: CELL-CYCLE CONTROLS SHUFFLE AND DEAL
}

\author{
Adèle L. Marston and Angelika Amon \\ Abstract | Meiosis is the type of cell division that gives rise to eggs and sperm. Errors in the \\ execution of this process can result in the generation of aneuploid gametes, which are \\ associated with birth defects and infertility in humans. Here, we review recent findings on how \\ cell-cycle controls ensure the coordination of meiotic events, with a particular focus on the \\ segregation of chromosomes.
}

\section{HOMOLOGUE}

One member of a chromosome pair (where each member of the pair is derived from one parent) in diploid organisms.

CYCLIN-DEPENDENT KINASE (CDK). A protein kinase that requires an associated cyclin protein for activity. Various CDK-cyclin complexes regulate different stages of the cell cycle.
Center for Cancer Research, Howard Hughes Medical Institute, Massachusetts Institute of Technology, E17-233, 40 Ames Street, Cambridge,

Massachusetts 02139, USA Correspondence to A.A. e-mail:angelika@mit.edu doi:10.1038/nrm1526
Meiosis is a specialized type of cell division that generates gametes with a haploid set of chromosomes. By contrast, mitosis produces daughter cells with a chromosome complement that is identical to that of the progenitor cell. The generation of haploid gametes requires both general cell-cycle regulators and meiosisspecific proteins. In this review, we will discuss how the mitotic cell-cycle machinery is modulated to bring about the meiotic programme, with particular emphasis on the modifications that result in the specialized meiotic chromosome-segregation programme (FIG. 1). Although the meiotic programme is not, strictly speaking, a cell cycle, in this review we will refer to the events from the decision to enter the meiotic programme up until the generation of gametes as the meiotic cell cycle, because many of the key regulators of the mitotic cell cycle control this process.

First, we will discuss the controls that regulate the decision to embark on either the meiotic or the mitotic cell cycle and how these regulatory pathways control the G1-S-phase transition. We will then compare and contrast the pre-meiotic and pre-mitotic $S$ phases. Next, we will summarize the aspects of meiotic G2 that are relevant to cell-cycle control, followed by a detailed discussion of the controls that bring about the unique pattern of chromosome segregation during meiosis I and leave in place the tools to segregate chromosomes during meiosis II. We will end with an overview of the regulatory circuits that control the specialized meiosis-I-meiosis-II transition and meiosis-II chromosome segregation. For some other aspects of the meiotic cell cycle, such as the pairing of
HOMOLOGUES, meiotic recombination and developmental signals that control progression through meiosis, the reader will be referred to recent reviews. Our discussion focuses primarily on studies that have been conducted in the fission yeast (Schizosaccharomyces pombe) and the budding yeast (Saccharomyces cerevisiae), and for this reason, yeast nomenclature is used, but for the corresponding gene and protein names in other organisms, please see TABLES 1,2.

\section{Regulation of the meiotic G1-S-phase transition}

The decision to enter the meiotic cell cycle occurs in response to cues that vary greatly among different organisms. In multicellular organisms, extrinsic cues from surrounding cells control the differentiation of germline stem cells that will enter the meiotic cell cycle. The molecular mechanisms that regulate this differentiation process are largely unknown ${ }^{1-3}$. In budding and fission yeast, poor nutrient conditions are the cue to embark on the meiotic cell cycle, which culminates in the production of spores ${ }^{4,5}$. The decision to enter the meiotic cell cycle is made in G1 phase and this affects the way in which the G1-S transition is controlled.

In budding yeast, nutrient limitation culminates in the expression of two principal regulators of meiotic initiation, inducer of meiosis (IME) 1 and IME2, which promote entry into pre-meiotic $S$ phase. These meiotic regulators substitute for the CYCLIN-DEPENDENT KINASE (CDK) Cdc28, which, when in complex with G1 cyclins (Clns), promotes entry into the mitotic cell cycle (FIG. 2). Ime1 is a transcription factor that initiates a 
a
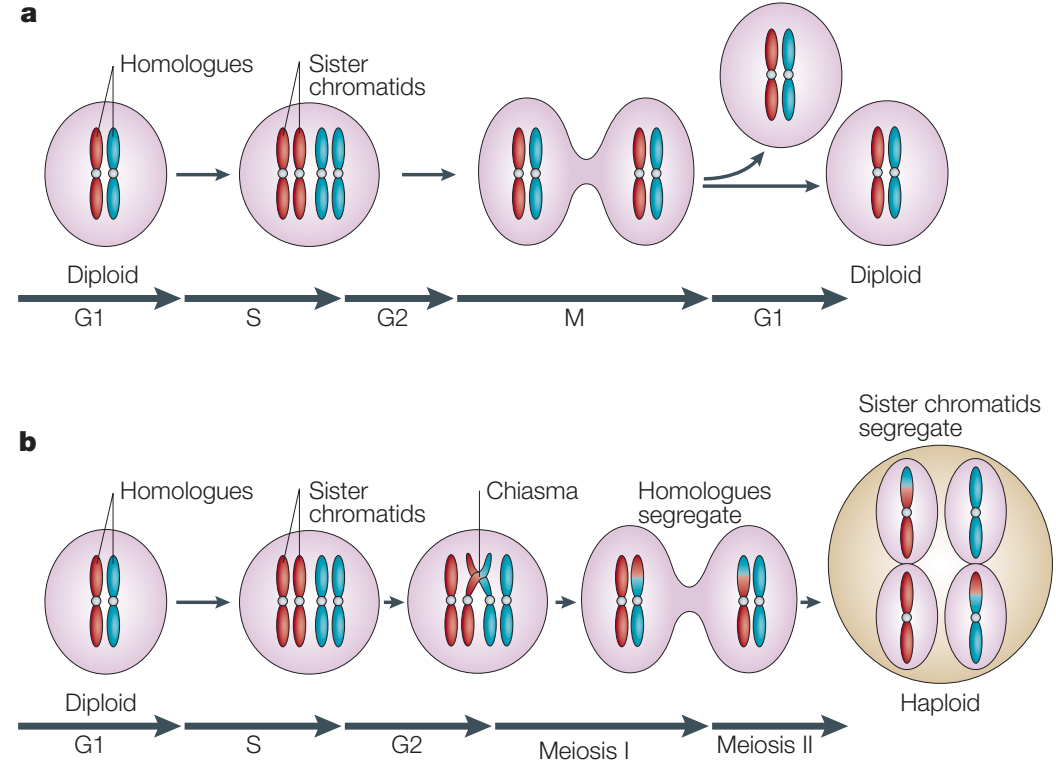

Figure 1 | The mitotic and meiotic cell cycles. a | In mitosis, diploid cells replicate chromosomes during S phase and segregate sister chromatids during M phase, so that diploid daughter cells are produced. $\mathbf{b}$ | In meiosis, two chromosome-segregation phases, meiosis I and meiosis II, follow a single round of DNA replication during pre-meiotic $S$ phase. During meiosis I, homologous chromosomes (shown in red and blue) are segregated to opposite poles. Sister chromatids segregate to opposite poles during meiosis II, which results in the formation of non-identical haploid gametes. Please note that the lengths of the cell-cycle stages are not drawn to scale.

\section{$\mathrm{APC} / \mathrm{C}$}

(Anaphase-promoting complex/cyclosome). A ubiquitin ligase which, together with a ubiquitin-conjugating enzyme, attaches ubiquitin peptides to a substrate protein. Ubiquitylated proteins are recognized by the $26 \mathrm{~S}$ proteasome and are subsequently degraded.

SYNAPTONEMAL COMPLEX (SC). A proteinaceous structure that forms between two homologues during meiotic G2, which is defined by a state of low CDK activity (when chromosomes are condensed cytologically speaking, this is prophase).

COHESIN

A protein complex that tethers sister chromatids together.

SISTER CHROMATIDS

Chromosomes that have been duplicated during $S$ phase.

MITOTIC/MEIOTIC SPINDLE A bipolar array of microtubules that forms during mitosis and meiosis to which chromosomes attach and by which

chromosomes are segregated to daughter cells. transcriptional programme that allows meiotic regulators to be generated in a temporally defined manner ${ }^{6,7}$. A key target of Ime1 is the IME2 gene, which encodes a meiosis-specific kinase with homology to CDKs. Ime2 promotes entry into pre-meiotic $S$ phase by carrying out some of the functions of Cln-CDKs (FIG. 2). Degradation of the S-phase CDK inhibitor, subunit inhibitor of CDK (Sic) 1, is dependent on IME2 (REF. 8). Ime2 also inhibits the ubiquitin-dependent proteolysis machinery that is defined by its ubiquitin ligase - the anaphase-promoting complex/cyclosome (APC/C) thereby allowing the stabilization of cyclin $\mathrm{B}(\mathrm{Clb})$ cyclins, which promote $S$ phase and chromosome segregation $^{9}$ (FIG. 2).

In fission yeast, entry into the meiotic cell cycle is prevented by the protein kinase Pat1. Pat1 blocks meiosis by phosphorylating the RNA-binding protein Mei2, the activation of which is, in itself, sufficient to initiate the meiotic programme $e^{10,11}$. In response to meiosis-inducing conditions, a specific Pat1 inhibitor, Mei3, is expressed - this releases the repression of Mei2 by Pat1 and initiates meiotic progression ${ }^{12,13}$. Mei2 cooperates with a specific small RNA, meiRNA ${ }^{14}$; however, the precise molecular function of Mei2 is unknown.

\section{Pre-meiotic S phase}

After cells have committed to the meiotic cell cycle, they undergo pre-meiotic $S$ phase. In budding yeast, premitotic as well as pre-meiotic DNA replication is triggered by S-phase CDKs, which are composed of the two B-type cyclins Clb5 and Clb6, and Cdc28 (REFS 8,15,16). Pre-meiotic DNA replication resembles pre-mitotic
$S$ phase in many respects. The same origins and replicative machinery are used and replication forks progress at similar rates ${ }^{17-20}$, at least in budding yeast. However, despite the usage of the same replicative machinery and regulators that control its activity during pre-mitotic and pre-meiotic DNA replication, there are likely to be differences between pre-mitotic and pre-meiotic DNA replication. In all organisms analysed so far, pre-meiotic $S$ phase is substantially longer than pre-mitotic $S$ phase ${ }^{21}$, and factors such as the budding yeast gene MUM2 have been found to be essential for pre-meiotic DNA replication, but not for pre-mitotic replication ${ }^{22}$. Lengthening of $S$ phase and the use of additional factors during pre-meiotic $S$ phase - in comparison with pre-mitotic $S$ phase - might be necessary because interactions between homologue pairs, which are necessary for their faithful segregation during meiosis I, are initiated during pre-meiotic $S$ phase.

Several studies in yeast have established a requirement for passage through $S$ phase to establish interhomologue interactions such as meiotic recombination $^{23,24}$, pairing of homologues ${ }^{24}$ and the formation of the SYNAPTONEMAL COMPLEX (SC $)^{24}$. Meiotic recombination begins with the deliberate introduction of DNA doublestrand breaks (DSBs) by the transesterase sporulation protein (Spo) 11 (REFS 25,26). This event requires DNA replication and occurs after bulk DNA synthesis ${ }^{23,27}$. Budding yeast $\mathrm{clb} 5 \mathrm{clb} 6$ mutants that fail to undergo bulk pre-meiotic DNA synthesis ${ }^{15}$ generate DSBs and SCs at levels that are proportional to the amount of DNA synthesis in these mutants ${ }^{24}$. Furthermore, when all origins of replication are deleted on the left arm of chromosome III, this chromosome arm replicates late during $S$ phase and DSB formation is delayed to a similar degree ${ }^{23}$. In fission yeast, however, mutants that fail to complete DNA synthesis were found to form DSBs at appreciable levels ${ }^{28}$. This could be because DSB formation is controlled differently in the two yeasts or, alternatively, it is possible that the observed DSBs correspond to the replicated part of the genome.

The requirement for passage through $\mathrm{S}$ phase for recombination and homologue pairing is explained by the fact that COHESIN, the protein complex that holds duplicated chromosomes (SISTER CHROMATIDS) together, has an essential role in these processes ${ }^{29,30}$. At least in fission yeast, cohesin must be laid down during $S$ phase to support inter-homologue interactions ${ }^{31}$. Although it has not been shown directly, there is likely to be a similar situation in budding yeast. Passage through pre-mitotic $S$ phase has been shown to be essential for cohesin to be functional ${ }^{32}$.

\section{Meiotic G2}

Meiotic G2 is defined by a state of low meiotic CDK activity. During this cell-cycle stage, linkages between homologue pairs are generated to ensure their co-alignment on the meiosis-I SPINDLE in preparation for segregation during meiosis I. For chromosomes of most, although not all, organisms, this linkage is brought about by at least one CHIASMA, which is generated as a result of meiotic recombination between the homologous 


\begin{tabular}{|c|c|c|c|c|c|c|c|c|}
\hline $\begin{array}{l}\text { Developmental } \\
\text { cycle }\end{array}$ & Sc & $S p$ & At & $\mathrm{Ce}$ & $D m$ & $X I$ & Mammals & References \\
\hline \multirow[t]{4}{*}{ Mitosis } & Scc1(Mcd1) & $\operatorname{Rad} 21$ & - & SCC-1/COH-2 & DRAD21 & XRAD21 & RAD21/HR21sp/PW29 & $\begin{array}{r}129-132,134 \\
166-171\end{array}$ \\
\hline & Scc3/lrr1 & Psc3 & - & SCC-3 & DSA & $\begin{array}{l}\text { XSA1 } \\
\text { XSA2 }\end{array}$ & $\begin{array}{l}\text { SA1/STAG1, } \\
\text { SA2/STAG2 }\end{array}$ & $\begin{array}{r}59,132,134,139,166 \\
167,169,170,178\end{array}$ \\
\hline & Smc1 & Psm1 & - & $\mathrm{HIM}-1 / \mathrm{SMC}-1$ & DSMC1 & XSMC1 & $\mathrm{SMC} 1 \alpha / \mathrm{mSMCB}$ & $\begin{array}{r}130-132,134,166,167 \\
169-172\end{array}$ \\
\hline & Smc3 & Psm3 & - & SMC-3 & DSMC3 & XSMC3 & SMC3/mSMCD & $\begin{array}{r}130-132,134,166,167 \\
169-172\end{array}$ \\
\hline \multirow[t]{4}{*}{ Meiosis } & $\operatorname{Rec} 8^{\ddagger}$ & $\operatorname{Rec} 8^{\ddagger}$ & SYN1-DIF1 ${ }^{\ddagger}$ & REC-8 ${ }^{\ddagger}$ & - & - & $\begin{array}{l}\text { REC8 }{ }^{\ddagger}, \\
\text { RAD21/SCC1 }\end{array}$ & $30,56-58,173-177,180$ \\
\hline & Scc3 & $\begin{array}{l}\text { Psc3§, } \\
\text { Rec11 } 1 \text { t\| }\end{array}$ & - & SCC-3 & - & - & $\begin{array}{l}\text { SA2/STAG2 } \\
\text { SA3/STAG3*\|1 }\end{array}$ & $59,139,174,178-180$ \\
\hline & Smc1 & Psm1 & - & SMC-1 & - & - & SMC1 $\beta^{\ddagger}$ & $57,58,180,181$ \\
\hline & Smc3 & Psm3 & - & SMC-3 & - & - & SMC3 & $30,57,58,130,180$ \\
\hline
\end{tabular}

*Potential cohesin complexes in mitosis and meiosis are shown based on the available functional or cytological data. ${ }^{\ddagger}$ Meiosis-specific proteins. ${ }^{\S}$ Proteins found to localize only to centromeric regions. "Proteins found to localize only to chromosome arms. At, Arabidopsis thaliana, Ce, Caenorhabditis elegans; COH-2, cohesin-2; Dm, Drosophila melanogaster; HR21sp, Homo sapiens Rad21; PW29, Pokeweed agglutinin-binding protein-29; Rec, recombination protein; Sc, Saccharomyces cerevisiae; Sp,

Schizosaccharomyces pombe; SAVSTAG, stromalin antigen; Scc, subunit of the cohesin complex; Smc, structural maintenance of chromosomes; XI, Xenopus laevis.

\section{CHIASMA}

(plural: chiasmata). Cytological manifestation of the point of exchange or crossing over

between two homologues due to meiotic recombination. chromosomes. We will only discuss the relevance of recombination to chromosome segregation and cellcycle progression (see REFS 33-35 for recent reviews on the mechanistic details of meiotic recombination).

Meiotic recombination is initiated upon the introduction of DSBs by Spo11 (REFS 25,26). These DSBs can be processed to generate two types of recombination product - either a crossover $(\mathrm{CO})$, in which reciprocal exchange between homologue pairs has occurred, or a non-crossover (NCO), in which reciprocal exchange has not occurred. Recent studies in budding yeast have shown the existence of two different recombination pathways for the processing of DSBs. One pathway generates only COs and an alternative pathway, predominantly generates $\mathrm{NCOs}^{36,37}$ (FIG. 3). The decision to generate a CO or an NCO is made soon after DSB formation ${ }^{35,37-39}$. Importantly, only COs result in the linking of homologue pairs. As these linkages are essential for the proper segregation of homologue pairs during meiosis I, elucidating the mechanisms that ensure the presence of at least one CO per pair of homologues will be crucial to understand how homologue segregation is regulated.

Recombination causes severe DNA damage. It is therefore essential that meiosis-I chromosome segregation does not proceed until all damage has been repaired. One or more surveillance mechanisms ensure that this is the case $\mathrm{u}^{40}$. The recombination, or pachytene, checkpoint is one such surveillance mechanism. It is activated concomitant with, or shortly after, DSB formation and delays entry into meiosis I until all DSBs have been repaired. The checkpoint is most well-characterized in budding yeast ${ }^{40,41}$ but is likely to exist in other organisms, as mouse spermatocytes and oocytes that are deficient for the recombination factor Dmc1, and therefore fail to repair DSBs, arrest in $\mathrm{G}^{42,43}$. The recombination checkpoint prevents entry into meiosis I by preventing meiotic CDK activation and the continuation of the developmental programme of meiosis (spore formation) by downregulating meiotic gene expression. CDKs are inhibited by the recombination checkpoint through activation of Saccharomyces Wee1 (Swe1), which phosphorylates Cdc28 on Tyr19, thereby inhibiting its activity ${ }^{44}$. Furthermore, transcription of the meiotic cyclins CLB1, CLB3 and CLB4 is prevented because the transcription factor $\mathrm{Ndt} 80$, which transcribes a large set of meiotic genes during G2, is inhibited, and the repressor of these genes, suppressor of Mar1 (Sum) 1, is active ${ }^{45-47}$. The downregulation of Ndt80dependent transcription also ensures that the developmental programme does not proceed, thereby establishing a complete G2 arrest until all DNA damage has been repaired.

The recombination checkpoint is not the only signal that halts meiotic cell-cycle progression in G2. In the female germline of most metazoans, developmental signals induce a G2 arrest. Oocytes arrest in G2 (diplotene) until their maturation is triggered during ovulation later in the life of the organism. The molecular mechanisms that control this cell-cycle arrest and the resumption of meiosis are complex and, in part, controversial, so we direct readers to reviews by experts in this field ${ }^{48,49}$.

\section{Meiosis I: a unique segregation event}

Once recombination has been completed, cells enter the meiotic divisions. Meiosis I is a unique type of chromosome-segregation event because it is the homologue pairs that segregate from each other, rather than the sister chromatids, as occurs in mitosis and meiosis II (BOX 1; FIG. 1). For this specialized segregation to occur and leave in place the tools to segregate sister chromatids during meiosis II, the mitotic chromosome-segregation machinery (summarized in BOX 1) must be modified in three ways (FIG. 4). First, homologue pairs must be linked, 


\begin{tabular}{|c|c|c|c|c|c|c|}
\hline Generic name & Sc & $S p$ & $\mathrm{Ce}$ & $X I$ & $D m$ & Mammalian \\
\hline $\begin{array}{l}\text { G1 cyclin-dependent- } \\
\text { kinase complex }\end{array}$ & $\begin{array}{l}\text { CDC28-CLN1, } \\
\text { CDC28-CLN2, } \\
\text { CDC28-CLN33 }\end{array}$ & cdc2-cig1, cdc2-puc1 & - & - & - & $\begin{array}{l}\text { Cdk4-cyclin-D, } \\
\text { Cdk6-cyclin-D, } \\
\text { Cdk2-cyclin-E }\end{array}$ \\
\hline $\begin{array}{l}\text { S-phase cyclin- } \\
\text { dependent-kinase } \\
\text { complex }\end{array}$ & $\begin{array}{l}\text { CDC28-CLB5, } \\
\text { CDC28-CLB6 }\end{array}$ & cdc2-cig2 & - & - & - & $\begin{array}{l}\text { Cdk2-cyclin-A, } \\
\text { Cdk2-cyclin-E }\end{array}$ \\
\hline $\begin{array}{l}\text { M-phase cyclin- } \\
\text { dependent-kinase } \\
\text { complex }\end{array}$ & $\begin{array}{l}\text { CDC28-CLB1, } \\
\text { CDC28-CLB2, } \\
\text { CDC28-CLB3, } \\
\text { CDC28-CLB4 }\end{array}$ & $c d c 2-c d c 13$ & - & - & - & $\begin{array}{l}\text { Cdk1-cyclin-B, } \\
\text { Cdk1-cyclin-A }\end{array}$ \\
\hline- & MIH1 & cdc25 & $C D C-25$ & $C D C 25$ & String & $\begin{array}{l}\text { CDC25A,CDC25B, } \\
\text { CDC25C }\end{array}$ \\
\hline- & SWE1 & wee1 & WEE-1 & WEE1 & WEE & WEE1 \\
\hline Separase & ESP1 & cut1 & $S E P-1$ & - & $\begin{array}{l}\text { Three Rows (THR), } \\
\text { Separase (SSE) }\end{array}$ & ESPL1 \\
\hline Securin & PDS1 & cut2 & $I F Y-1$ & PTTG & Pimples (PIM) & PTTG1 \\
\hline- & SPO11 & rec12 & SPO-11 & - & MEI-W68 & SPO11 \\
\hline Shugoshin & SGO1 & sgo 1 & - & - & MEI-S332 & - \\
\hline Polo kinase & CDC5 & plo1 & PLK-2 & $P L X 1$ & POLO & PLK1 \\
\hline Aurora kinase & $\begin{array}{l}- \\
\text { IPL1 } \\
-\end{array}$ & $\begin{array}{l}- \\
\text { ark1 } \\
-\end{array}$ & $\begin{array}{l}\text { AlR-1 } \\
\text { AlR-2 } \\
-\end{array}$ & $\begin{array}{l}\text { Aurora- } A(E g 2) \\
\text { Aurora- } B \\
-\end{array}$ & $\begin{array}{l}\text { Aurora-A } \\
\text { Aurora-B } \\
-\end{array}$ & $\begin{array}{l}\text { Aurora- } A \\
\text { Aurora- } B \\
\text { Aurora- } C\end{array}$ \\
\hline- & CDC14 & clp1 & $C D C-14$ & - & - & $C D C 14 A, C D C 14 B$ \\
\hline- & MAD2 & $\operatorname{mad} 2$ & MDF-2 & MAD2 & MAD2 & MAD2L1, MAD2L2 \\
\hline
\end{tabular}

AIR, Aurora/Ipl1-related kinase; ark1, aurora kinase-1; Cdk, cyclin-dependent kinase; Ce, Caenorhabditis elegans; CLB, cyclin B; CLN, cyclin; clp1, Cdc14-related protein phosphatase-1: Dm Drosophila melanogaster; ESP1, extra spindle poles-1; ESPL1, extra spindle poles-like-1; IFY-1, interactor of FZY-1; MAD2, mitotic arrest-deficient-2; MDF-2, yeast mitosis-arrest-deficient related-2; MIH1, mitotic inducer homologue-1; PDS1, prevents the dissociation of sisters-1; PLK/plo/PLX/POLO, polo kinase; PTTG, pituitary tumor-transforming protein; Rec, recombination protein; Sc, Saccharomyces cerevisiae; SEP-1, separase-1; Sp, Schizosaccharomyces pombe; SGO1; shugoshin-1; SPO, sporulation protein; Swe1, Saccharomyces Wee1; XI, Xenopus laevis.

COHESION

The sticking together of two sister chromatids.

\section{SPINDLE POLE}

The yeast equivalent of the centrosome that nucleates microtubules, including those that will form the spindle.

\section{BIVALENT}

A pair of homologues that are linked together following meiotic $\mathrm{G} 2$, which is defined by a state of low CDK activity (when chromosomes are condensed cytologically speaking, this is prophase).

\section{SEPARASE}

An enzyme that cleaves the cohesin subunit $\mathrm{Sccl}$ or Rec8 during mitosis and meiosis. usually by chiasmata, to ensure their alignment on the meiosis-I spindle. Second, some linkage (COHESION) between sister chromatids must be maintained beyond meiosis I to prevent their premature dissociation and ensure their proper attachment to the meiosis-II spindle. Third, sister chromatids have to attach to microtubules that emanate from the same SPINDLE POLE in meiosis I, but from opposite poles in meiosis II.

Chiasmata hold homologues together. Chiasmata are generated as a result of reciprocal recombination between homologue pairs, and they hold the pairs together. This allows the BIVALENT (a pair of recombined homologues) to align correctly on the meiosis-I spindle (FIG. 4). The importance of chiasmata for accurate meiosis-I chromosome segregation has been shown by the observation that budding yeast and nematode worm (Caenorhabditis elegans) mutants that lack SPO11 — and therefore do not initiate meiotic recombination - randomly segregate their homologues at meiosis $\mathrm{I}^{26,30,50}$. In fission yeast, the inactivation of the Spo11 homologue, Rec12, results in near-random segregation at meiosis I (REF. 51), and Spo11 is also essential to generate functional gametes in the mouse $^{52,53}$. Sister-chromatid cohesion on chromosome arms, distal to chiasmata, stabilizes the homologue interactions that are mediated by chiasmata. As described below, the loss of chromosome-arm cohesion is the single event that is required to allow homologues to segregate from each other during meiosis I.

\section{Meiosis I: loss of arm cohesion}

As mentioned above, sister chromatids, which are generated during $S$ phase, are held together by a protein complex that is known as cohesin, which is thought to form a ring around the duplicated $\mathrm{DNA}^{54}$. During the mitotic as well as the meiotic cell cycle, cohesins must be assembled onto the DNA during DNA replication for them to function as cohesion factors ${ }^{31,32}$ (BOX 1). During mitosis, cleavage of one of the cohesin subunits, Scc1(Mcd1)/Rad21 (the budding yeast protein subunit of the cohesin complex ( $\mathrm{Scc}$ ) 1 is encoded by the MCD1 gene; $\operatorname{Rad} 21$ is the Scc1 homologue in fission yeast), by a protease that is known as SEPARASE initiates mitotic chromosome segregation (BOX 1).

During meiosis, the existence of two consecutive rounds of chromosome segregation requires that cohesion between sister chromatids is lost in a stepwise manner. Loss of cohesion on chromosome arms in meiosis I abolishes the linkage between homologue pairs and allows them to separate to opposite poles of the meiosis-I spindle. Cohesion between sister chromatids is, however, maintained around the centromeric regions to ensure that they do not drift apart before anaphase II and to promote the proper attachment of sister chromatids to the meiosis-II spindle. This stepwise loss of cohesion requires some changes to the cohesin complex itself and the way it is regulated.

A conserved modification of the meiotic cohesin complex is the substitution of the $\operatorname{Scc} 1(\operatorname{Mcd} 1) / \operatorname{Rad} 21$ 


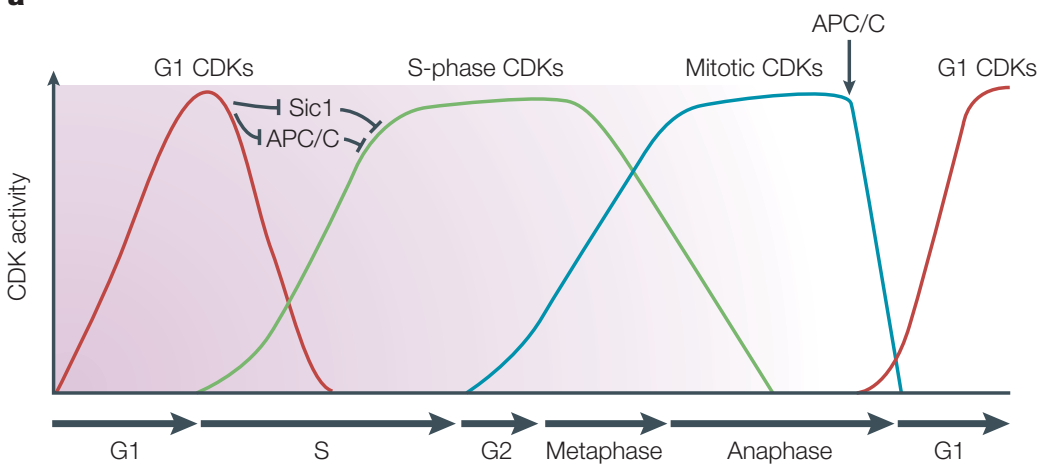

b

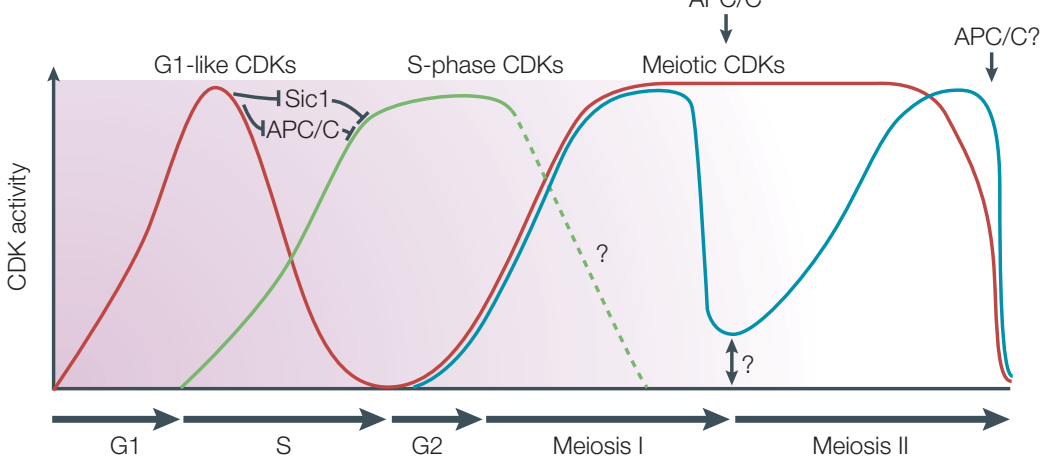

Figure 2 | Cyclin-dependent-kinase activity in meiosis and mitosis. a | During G1, G1 cyclin-dependent kinase (CDK) activity (red) rises and induces the destruction of Sic1 and the inactivation of the anaphase-promoting complex/cyclosome (APC/C), thereby allowing entry into the cell cycle and the accumulation of S-phase CDK activity (green). S-phase CDKs initiate DNA replication ${ }^{163,164}$. Mitotic CDKs (blue) promote entry into mitosis. At the end of mitosis, mitotic CDKs are inactivated, which allows for the disassembly of the mitotic spindle and entry into G1. Inactivation of mitotic CDKs occurs through B-type-cyclin destruction ${ }^{165}$. $\mathbf{b} \mid$ During the meiotic cell cycle, a G1-like CDK (red; Ime2 in budding yeast) controls entry into the cell cycle and promotes the activation of S-phase CDKs (green; Cdc28-cyclin-B-5/6 (Clb5/6) in budding yeast) by inducing Sic1 destruction and inactivation of the APC/C. Ime2 has a second peak in kinase activity during the meiotic divisions and is required for the execution of the meiotic divisions ${ }^{16}$. It is not known if S-phase CDK activity declines after entry into meiosis I (dotted green line) $)^{77}$. Meiotic CDKs (blue; Cdc28-Clb1/3/4 in budding yeast) direct chromosome segregation during meiosis I. In the frog (Xenopus laevis), meiotic CDKs are partially inactivated between meiosis I and meiosis II, which prevents further DNA replication and chromosome segregation ${ }^{113}$. Meiotic CDK activity rises again to allow entry into meiosis II. Complete inactivation of meiotic CDKs triggers exit from meiosis II. Please note that the lengths of the cell-cycle stages are not drawn to scale.

\section{CENTROMERE}

The region of the DNA on which the kinetochore assembles.

HOLOCENTRIC CHROMOSOME A chromosome that has centromeres distributed along its length, which are known as diffuse centromeres.

MONOCENTRIC CHROMOSOME A chromosome with a single centromere.

\section{SECURIN}

An inhibitor of separase that keeps the protease inactive until the onset of anaphase, at which point securin is destroyed, thereby liberating separase. subunit with a meiosis-specific variant, Rec8 (TABLE 1). Rec8 is essential for sister-chromatid cohesion during meiosis in budding yeast, fission yeast and nematode worms $\mathrm{s}^{30,31,55}$. Immunolocalization experiments showed that Rec8 is lost from chromosome arms during meiosis I, but retained around CENTROMERES until meiosis II in yeast ${ }^{30,56}$, mice $^{57}$ and rats $^{58}$. In nematode worms, in which the chromosomes are HOLOCENTRIC during mitosis, but become functionally MONOCENTRIC during meiosis, an equivalent, stepwise loss of cohesin occurs. In this organism, Rec8 is partially lost during meiosis I, but retained between the facultative centromere and the nearest crossover until meiosis II (REF. 55).

In some organisms, there are several different cohesin complexes, of which some are specific to meiosis (TABLE 1). In fission yeast meiosis, the mitotic Psc3 subunit is found in cohesin complexes around centromeres but is replaced on chromosome arms with a meiosis-specific variant, Rec11 (REF. 59) (TABLE 1). This specialization of arm cohesin could contribute to the differential timing with which arm and centromeric cohesins are lost in meiosis. Furthermore, Rec11, like Rec8, has a role in meiotic recombination that cannot be fulfilled by its mitotic counterpart ${ }^{30,60,61}$. Perhaps these cohesin-subunit variants promote inter-homologue invasion during recombination rather than invasion into the sister chromatid (which is the way DSBs are repaired during the mitotic cell cycle). They might thereby facilitate the generation of chiasmata, which is essential for meiosis-I chromosome segregation.

\section{Meiosis I: regulators of cohesin loss}

The role of separase. One possible mechanism to allow for the differential loss of arm and centromeric cohesin would be to restrict separase activity to meiosis II and remove cohesins from chromosome arms in a separaseindependent manner during meiosis I, which is akin to the non-proteolytic way in which cohesins are removed from chromosome arms during prophase in mammalian cell $^{62,63}$ (BOX 1). However, elegant experiments in budding and fission yeast showed that separase-dependent cleavage of Rec8 triggers the loss of cohesins, and therefore chromosome segregation during meiosis I and meiosis II (REFS 64,65). Whether the loss of cohesin by separase cleavage mediates the loss of arm cohesion in meiosis I and II in other eukaryotes is not known. However, a function for separase and its regulator, the ubiquitin ligase APC/C, in the segregation of homologues during meiosis I has also been shown in nematode worm ${ }^{66,67}$ and mouse oocytes $^{68,69}$, which indicates that separase-dependent cleavage of Rec8 on chromosome arms is conserved. Curiously, however, destruction of the separase inhibitor SECURIN by the APC/C does not seem to be required for meiosis-I chromosome segregation in frog (Xenopus laevis) oocytes ${ }^{70,71}$, which suggests that different controls may remove cohesins from chromosome arms in this organism.

A role for Rec 8 . The fact that separase controls the loss of cohesion during both meiosis I and meiosis II, at least in budding and fission yeast, predicts that cohesin complexes around centromeres must be resistant to cleavage by separase during meiosis I. Factors that 'protect' centromeric Rec8 from cleavage have to be present during meiosis I but removed before, or at, the onset of anaphase II. One important factor in the protection of centromeric cohesins is Rec8 itself. The replacement of Rec8 with $\operatorname{Scc} 1(\operatorname{Mcd} 1) / \operatorname{Rad} 21$ leads to the loss of cohesins along the entire length of chromosomes at the onset of anaphase I in budding and fission yeast ${ }^{72,73}$. This indicates that a property of Rec 8 that is not shared with $\operatorname{Scc} 1(\operatorname{Mcd} 1) / \operatorname{Rad} 21$ is important for its protection at centromeres. One difference between Scc1 (Mcd1) and Rec 8 in budding yeast is their relative dependence on the Polo-like kinase Cdc5 for cleavage by separase. Phosphorylation of Scc1 (Mcd1) by Cdc5 facilitates Sccl(Mcd1) cleavage, but is not essential ${ }^{74}$. By contrast, 


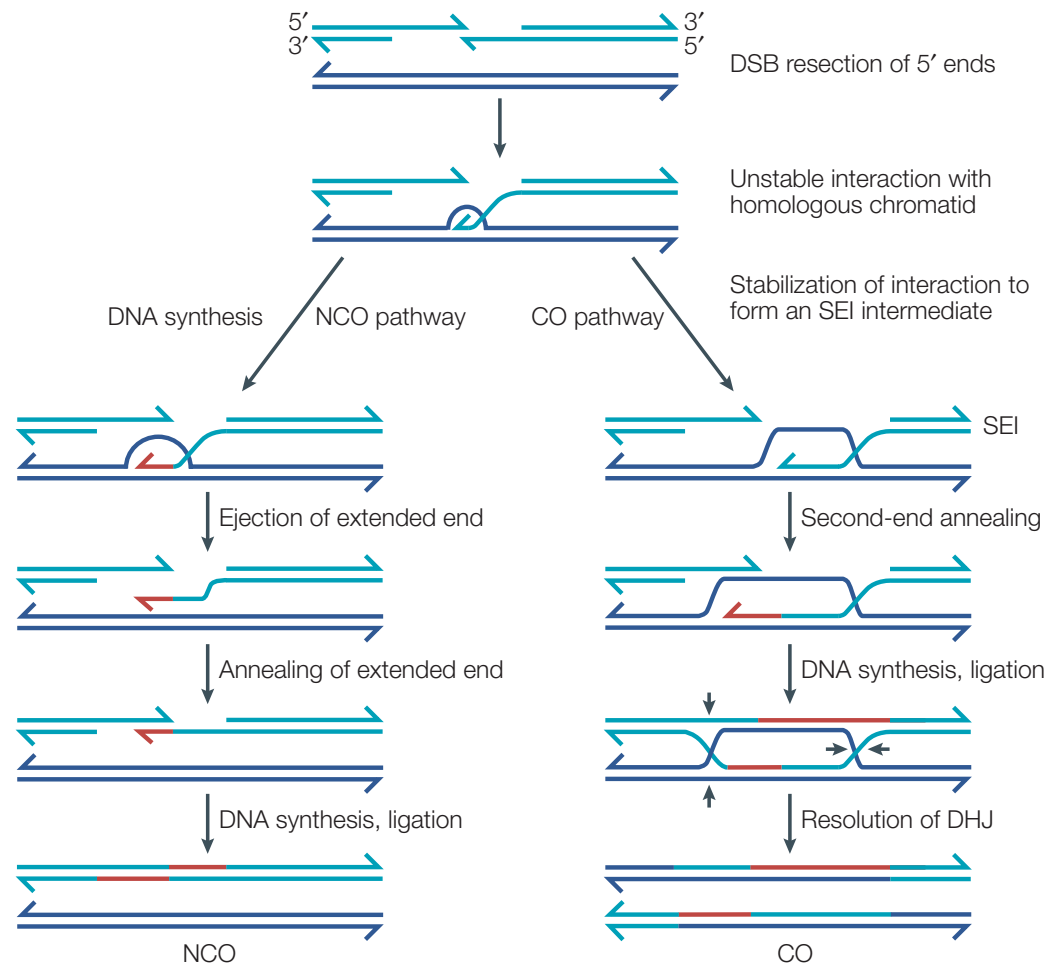

Figure 3 | The early crossover decision (ECD) model for meiotic recombination.

Recombination initiates with DNA double-strand breaks (DSBs). DNA cleavage is mediated by the highly conserved sporulation protein (Spo) 11 topoisomerase. Following DSB formation, the DNA is resected from its $5^{\prime}$ end, a reaction that depends on a complex that comprises Rad50, meiotic recombination protein (Mre)11 and X-ray-sensitive protein (Xrs)2. Next, $3^{\prime}$ single-stranded DNA tails invade the intact homologous DNA duplex. This nascent $3^{\prime}$ interaction is unstable and it is at this step of the process that the decision is made to follow the non-crossover (NCO; left) or the crossover (CO) pathway (right). In the NCO pathway, the 3' single-stranded tail initiates DNA synthesis (shown in red) but the extended end is ejected. It then anneals with its partner and DNA synthesis is completed and followed by ligation. In the CO pathway, the nascent $3^{\prime}$ interaction is stabilized to form a single-end invasion (SEI) intermediate. The second $3^{\prime}$ end then invades or anneals with the displaced strand of the SEl intermediate (second-end annealing). DNA synthesis then ensues from both $3^{\prime}$ ends and is followed by ligation to form a double Holliday junction (DHJ). The DHJ is then nicked as indicated by the arrows and resolved to form two recombinant DNA molecules. Adapted from REF. 35.

KINETOCHORE

A complex that is composed of a large number of proteins that mediate the attachment of chromosomes to microtubules.
Rec8 is absolutely dependent on Cdc5 for cleavage $\mathrm{e}^{75,76}$, although it is not known whether Cdc5-dependent phosphorylation of Rec8 is important for cleavage. One attractive hypothesis is that $\mathrm{Cdc} 5$ is inhibited from phosphorylating cohesins around the centromere, which leaves Rec 8 refractory to cleavage by separase in this region. Substituted Scc1 $(\operatorname{Mcd} 1)$ could, however, still be cleaved as its dependence on Cdc5 phosphorylation is not absolute ${ }^{74,76}$. Alternatively, Rec8, but not Scc1 $(\operatorname{Mcd} 1)$, might be able to interact with proteins around centromeres that result in Rec 8 being resistant to cleavage by separase.

MEI-S332 and other factors. Several factors, in addition to Rec8 itself, have been identified that control the stepwise loss of cohesins from chromosomes. In budding yeast, the meiosis-specific protein Spo13, which bears no conserved motifs, is present during meiosis I, but not meiosis II (REF. 77), and functions in the maintenance of centromeric cohesion. Cells that lack SPO13 are impaired, although not completely deficient, in their ability to retain Rec8 at centromeres during meiosis I (REF.30) and, when overproduced, Spo13 can prevent cleavage of Rec8 or Scc1 (Mcd1), despite the fact that separase is active in these cells $s^{78,79}$. These results imply that Spo13 prevents the loss of centromeric cohesion; however, the question still remains how the region around the centromere is singled out for protection in meiosis I.

The fruitfly (Drosophila melanogaster) protein MEIS332 is ideally situated to function as a protein that prevents cohesin removal around centromeres during meiosis I. MEI-S332 localizes around centromeres but dissociates from these chromosomal regions at anaphase II, which corresponds to the time at which centromeric cohesion is lost in meiosis II (REF. 80,81). More importantly, in mei-S332 mutants, sister-chromatid cohesion is lost prematurely ${ }^{82}$. Recently, three different genetic screen $s^{83-85}$ identified a coiled-coil protein, shugoshin (also known as Sgo1), that is distantly related to MEI-S332. Subsequently, Sgol homologues have been identified in almost all eukaryotes ${ }^{84,86}$. Like MEI-S332, budding yeast Sgol associates with KINETOCHORES from G2 until metaphase II (REFS 83,85). In fission yeast, however, most Sgol dissociates from centromeric regions during anaphase I (REFS 84,86). The reason for this difference in behaviour is, at present, unclear. In sgo1 $\Delta$ mutants of both yeasts, however, Rec8 is not retained at centromeric regions during meiosis I, and chromosomes segregate randomly at meiosis II (REFS 83-85). A key question is whether Sgol is required for the ability of the cohesin complex to establish cohesion at the centromere, or whether Sgol functions as a 'protector' of cohesin, which ensures that cohesion is not lost until meiosis II. At least in fission yeast, Rec8 must be cleavable by separase to allow sister chromatids to separate at meiosis II in an sgol $\Delta$ mutant $^{86}$. This finding suggests that cohesion is established at the centromere in the absence of SGO1, and that SGO1 regulates cohesion by preventing premature separase-dependent cleavage of Rec 8 around centromeres. Elucidating the mechanism by which Sgol does this remains an important challenge for the future. Sgol could prevent access of separase to substrates or could prevent phosphorylation of Rec 8 by Cdc5, which would make Rec8 resistant to cleavage.

Interestingly, MEI-S332 proteins also seem to function during mitosis. MEI-S332 is present during mitosis and might contribute to sister-chromatid cohesion in mitosis ${ }^{87}$. Fission yeast Sgo 1 does not function in mitosis, but a second Sgo protein, which is known as Sgo2, is required for accurate chromosome segregation in mitosis and, curiously, meiosis I but not meiosis II (REFS 84,86). In budding yeast, Sgo 1 is also present at kinetochores during mitosis until the onset of anaphase and has an undefined role in chromosome segregation ${ }^{83-85}$. Although it is present during mitosis, Sgol does not prevent cohesin removal from centromeric regions during anaphase. This is probably due to the dissociation of Sgol from kinetochores at the onset of anaphase ${ }^{83-85}$. Therefore, 
during meiosis, other factors, for example, Spo13like molecules, must contribute to the ability of Sgo1 to protect centromeric cohesion during meiosis I. Perhaps the key function of such meiosis-specific factors is to inhibit the removal of Sgol from kinetochores during meiosis I, in which case it will be critical

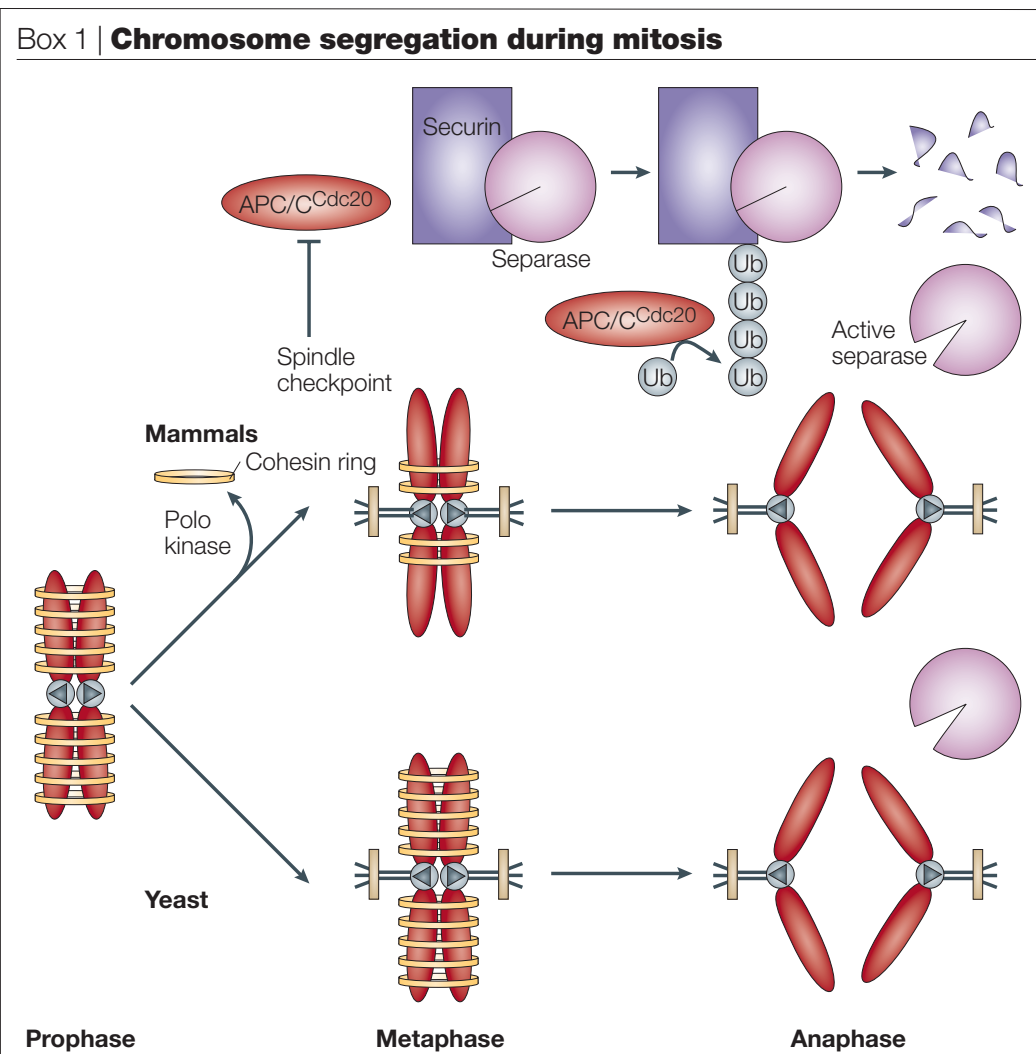

The alignment of chromosomes on the metaphase spindle and their subsequent segregation at anaphase depends on the establishment and dissolution of linkages between sister chromatids. In mitosis, the cohesin complex, which consists of four proteins (structural maintenance of chromosomes $(\mathrm{Smc}) 1, \mathrm{Smc} 3$, subunit of the cohesin complex $(\mathrm{Scc}) 1(\mathrm{Mcd} 1) / \operatorname{Rad} 21$ and Scc3/Psc3 (the mammalian homologues of which are SA1/SA2)), provides this sister-chromatid cohesion ${ }^{129-134}$ (TABLE 1). A fifth protein, precocious dissociation of sisters (Pds)5, is also essential for cohesin function ${ }^{132,135-139}$. Other factors function in the generation of cohesion by promoting the assembly of cohesins on the DNA. First, deposition factors load cohesins onto DNA. Subsequently, for these cohesins to be functional, other factors promote the capture of the two sister chromatids, presumably within the cohesin ring ${ }^{32}$.

For sister chromatids to be separated at anaphase, cohesion is eliminated by the proteolytic cleavage of $\operatorname{Scc} 1(\mathrm{Mcd} 1) / \operatorname{Rad} 21$ by the protease separase (Esp1 in budding yeast or Cut 1 in fission yeast $)^{140-142}$. Separase is held inactive until the onset of anaphase through its binding to the inhibitor protein securin (Pds1 in budding yeast or Cut2 in fission yeast). Activation of separase occurs when the anaphase-promoting complex/cyclosome (APC/C), together with $\mathrm{Cdc} 20$, targets securin for destruction by the proteasome $^{143-145}$ (see figure). In mammalian cells, the bulk of cohesin is removed from chromosome arms during prophase in a separase-independent, but Aurora-B- and Polokinase-dependent, manner ${ }^{62,63}$. However, a pool of cohesins is retained on chromosomes, particularly around centromeres, which is sufficient to hold sister chromatids together. Sister chromatids are allowed to separate during anaphase only when separase-mediated cleavage of Sccl occurs ${ }^{142}$. The spindle checkpoint prevents anaphase onset when kinetochores are not attached to the mitotic spindle ${ }^{146}$. The checkpoint components $\mathrm{Mad} 2$ and a BubR1-containing complex bind to the $\mathrm{APC} / \mathrm{C}^{\mathrm{Cd} 220}$, which renders the ubiquitin ligase inactive and prevents separase activation. to understand how Sgo 1 association with kinetochores is regulated.

\section{Meiosis l: centromeric cohesion}

Some kinetochore proteins are important for defining the domain of centromeric cohesion that is protected during meiosis I (REFS 88-90). Fission yeast protein Bub1, a protein kinase with a conserved function in the spindle checkpoint (a checkpoint that monitors the attachment of microtubules to kinetochores; see BOX 1), is required for the retention of Rec8 at centromeres during meiosis I (REF. 91). This requirement can be explained by the failure of Sgol to localize to kinetochores in the bub1 $\Delta$ mutant $^{84}$. In budding yeast, two kinetochore proteins, increased minichromosome loss $(\mathrm{Iml}) 3$ and chromosome loss $(\mathrm{Chl}) 4$, are required for the maintenance of centromeric cohesion in meiosis I (REF. 85). However, not all kinetochore proteins are involved in regulating centromeric cohesion ${ }^{85}$, which argues that Iml3 and Chl 4 have a direct role in the establishment of cohesion, rather than simply protecting the integrity of the kinetochore.

It is also clear that Rec8 is not protected from removal solely at the centromere, but is also protected on the adjacent chromatin. Cohesins are also maintained at pericentromeric heterochromatin, which is required to preserve cohesion between sister chromatids beyond meiosis I in organisms other than budding yeast (budding yeast does not have pericentromeric heterochromatin; FIG. 5 $)^{92,93}$. In fission yeast, the recruitment and maintenance of Rec8-Psc3 cohesin complexes to pericentromeric heterochromatin depends on the heterochromatin-establishment factors Swi6 and cryptic loci regulator (Clr) 4 (REF. 59) (FIG. 5). However, the retention of Rec8-Psc3 complexes at the central core of the fission yeast centromere is independent of Clr4 or Swi6. This indicates that cohesin complexes localize to centromeres and pericentromeric regions through different mechanisms $s^{59}$. Sgol is localized to the pericentromeric regions, rather than the central-core regions, so it is ideally situated to protect precisely those Rec8 complexes that preserve cohesion around centromeres ${ }^{84}$. In the fruitfly, MEI-S332 localization depends on functional centromeric chromatin but is separable from kinetochore assembly ${ }^{94,95}$. How can we reconcile the observations that pericentromeric heterochromatin and bona fide kinetochore proteins are both important for the establishment of a domain where cohesins are protected from removal? Perhaps in analogy to the ability of the kinetochore to organize a large domain of cohesion around itself ${ }^{96}$, the kinetochore functions as a seed from which the protective proteins are spread throughout the pericentromeric region.

\section{Meiosis I: kinetochore co-orientation}

During mitosis, sister chromatids attach to microtubules from opposite poles and are segregated away from each other before cell division (FIG. 1). In mitosis and meiosis II, kinetochores of sister chromatids (sister kinetochores) 

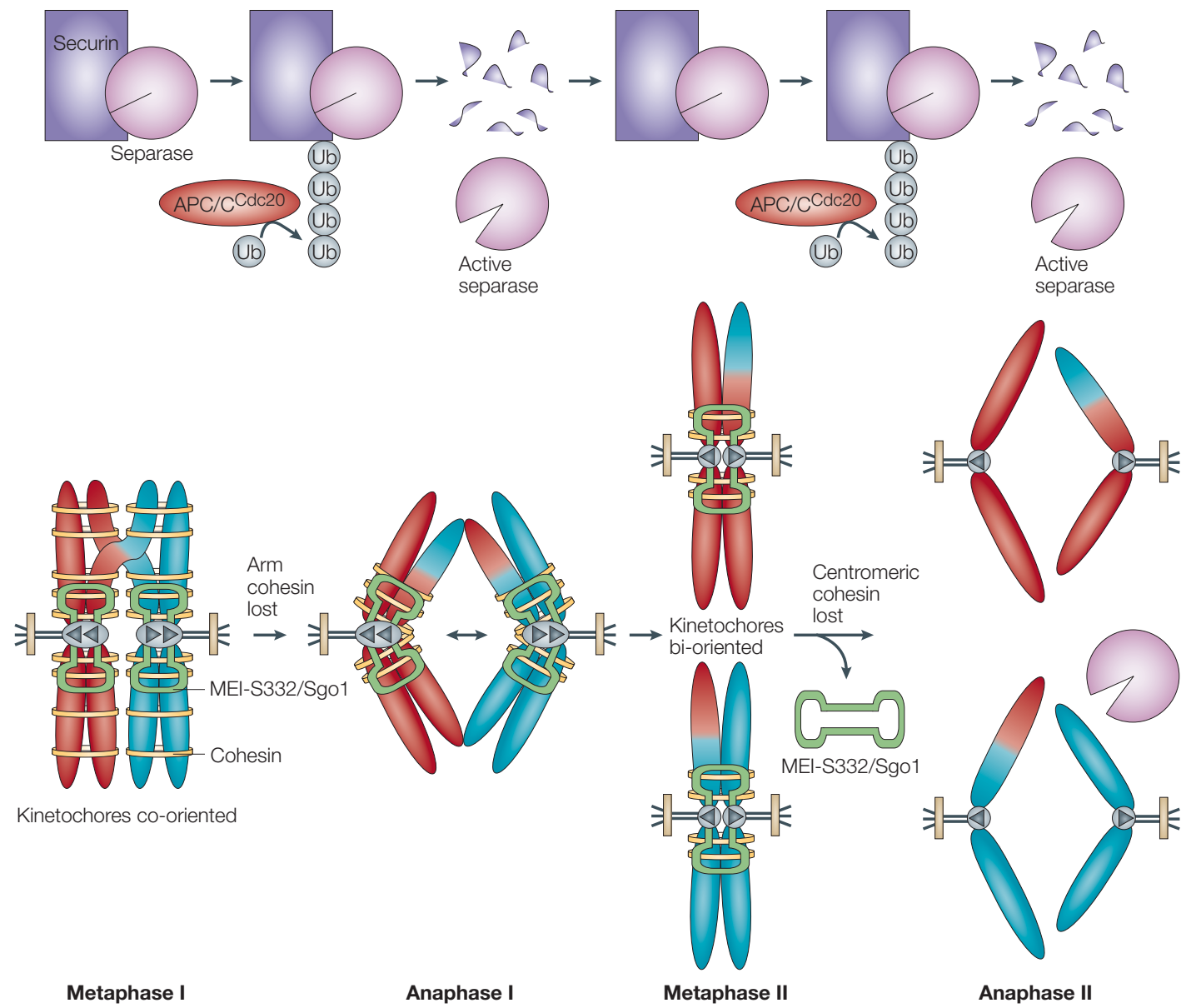

Figure 4 | A model for meiotic chromosome segregation. Three factors contribute to the segregation of homologues at meiosis I. First, homologues (shown in red and blue) are linked by at least one chiasma. Second, kinetochores attach to microtubules that emanate from the same pole (co-orientation; indicated by the direction of the arrows on the sister kinetochores). Third, sister chromatids are held together by cohesin rings (yellow). MEI-S332/Sgo1 (green) is shown around the centromere. During metaphase I, chromosomes are aligned ready for segregation, but separase (pink) is kept inactive by securin (purple). At the onset of anaphase I, $\mathrm{APC} / \mathrm{C}^{\mathrm{Cd} 202}$ (red) becomes active and ubiquitylates securin, thereby targeting it for destruction. Active separase now cleaves the Rec8 subunit of cohesin on the chromosome arms, which triggers the separation of homologues to opposite poles of the meiosis-I spindle. Centromeric Rec8 is protected from cleavage, perhaps due to the presence of MEI-S332/Sgo1. At metaphase II, kinetochores are bi-oriented and separase is once again inhibited by securin. MEI-S332/Sgo1 dissociates concomitant with, or before, the re-activation of separase at the metaphase-II-anaphase-II transition. Finally, this MEI-S332/Sgo1 dissociation leaves centromeric Rec8 free for cleavage by separase, which triggers the segregation of sister chromatids to opposite poles. Note that in fission yeast, Sgo1 dissociates from kinetochores before metaphase II and in the frog (Xenopus laevis) removal of cohesin in meiosis I seems to be separase independent ${ }^{71}$. Ub, ubiquitin.

\section{AMPHITELIC}

Connection of sister

kinetochores to microtubules that emanate from opposite spindle pole bodies.

SYNTELIC

Connection of sister kinetochores to microtubules that emanate from the same spindle pole body. must attach to microtubules from opposite poles - a situation that is known as bi-orientation or AMPHITELIC attachment (FIG. 4). In meiosis I, however, sister kinetochores uniquely attach to microtubules from the same pole to ensure their co-segregation (FIGS 4,6). This is known as monopolar attachment, and sister kinetochores are said to be mono-oriented (also, more recently, referred to as co-oriented ${ }^{97}$ ) or to have sYNTELIC attachment. The molecular mechanisms for this important modification in kinetochore attachment are beginning to be understood.

Cytological observations in several species indicated that sister kinetochores are fused into a single unit during meiosis I at the time of microtubule attachment, but they resolve into two distinct structures before the onset of anaphase-I chromosome segregation ${ }^{98,99}$. In addition, functional studies indicated that it is a property of the chromosome, rather than the microtubules or the cellcycle state, that ensures co-orientation of kinetochores in meiosis I. Paliulis and Nicklas ${ }^{100}$ found that a homologue pair that was taken from grasshopper cells in meiosis I segregates in a meiosis-I-like manner when introduced onto a meiosis-II spindle by micromanipulation ${ }^{100}$. These observations imply that the kinetochore is modified in meiosis I to ensure the co-orientation of sister chromatids.

Which chromosomal events are important for sisterkinetochore co-orientation? A physical linkage between homologues does not seem to be important for the monopolar attachment of sister kinetochores, at least in budding and fission yeast. Abolition of the linkages 


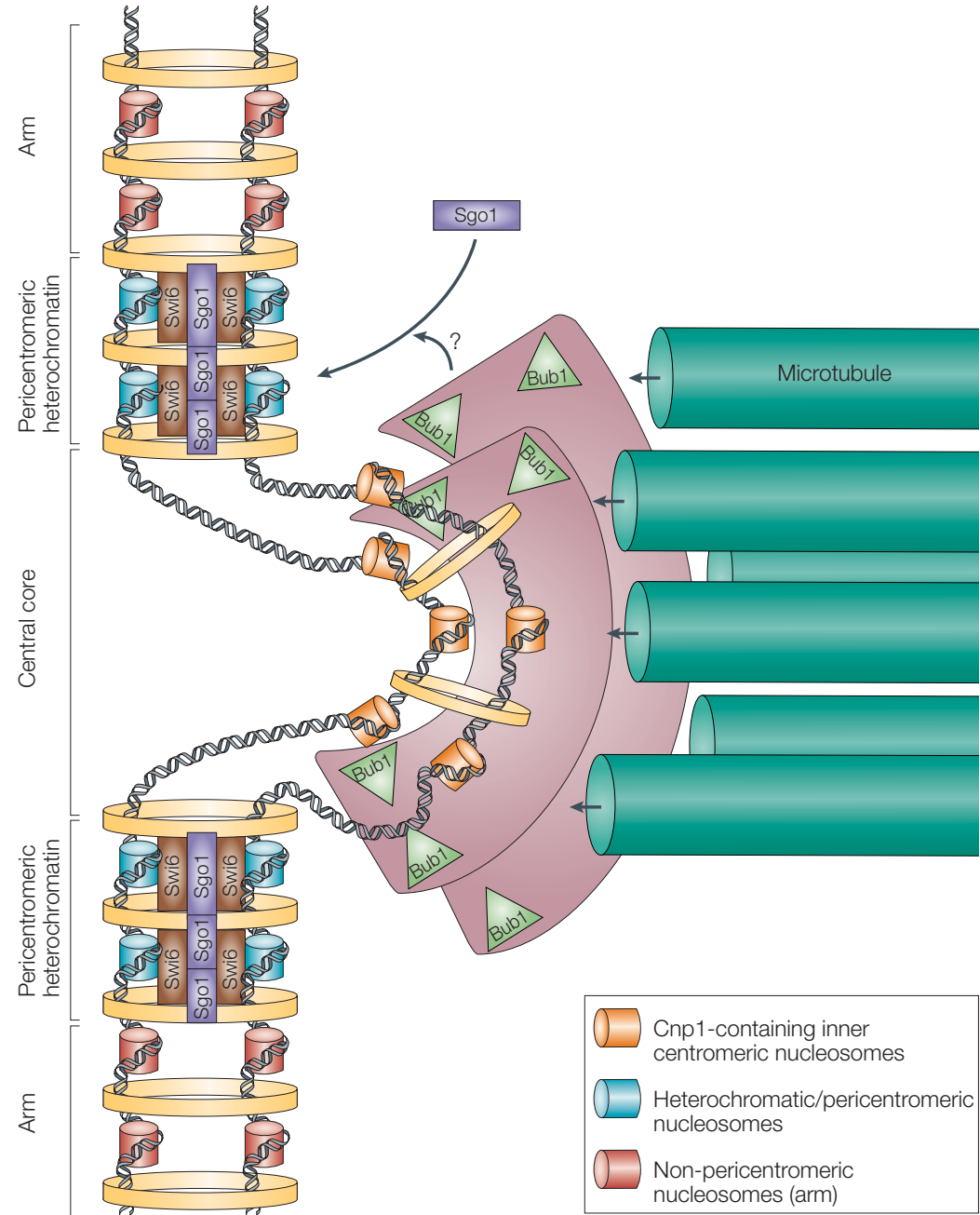

Figure $5 \mid$ A model for the control of pericentromeric and centromeric cohesion in fission yeast. Sister chromatids of one homologue are held together by cohesin rings (yellow).

Specialized nucleosomes that contain the histone variant centromere protein-1 (Cnp1; the fission yeast orthologue of human CENP-A; orange) are present at the central-core regions. Outside the centromeric and pericentromeric regions, on the chromosome arms, nucleosomes are hypoacetylated (red), whereas the pericentromeric region is heterochromatic and nucleosomes are acetylated (blue). These heterochromatic nucleosomes attract the heterochromatinestablishment factor, Swib. The inner centromere directs the assembly of the kinetochore, which mediates the binding of microtubules (dark green). We postulate that binding of shugoshin (Sgo1), and perhaps other cohesin protectors, to the pericentromeric region is controlled both by heterochromatin-establishment factors and bona fide kinetochore proteins. The precise location of Bub1 at the centromere is unknown. Adapted from REFS 31,59.

MONOPOLIN COMPLEX

A protein complex that ensures the syntelic attachment of sister kinetochores during meiosis I.

\section{MEROTELIC}

Attachment of a single

kinetochore to microtubules

from both spindle-pole bodies. between homologues (in spo11 $1 \Delta$ or rec $12 \Delta$ mutants) does not significantly interfere with sister-kinetochore co-orientation ${ }^{30,59,101}$, but Rec12 of fission yeast might become important for monopolar attachment in situations where kinetochore orientation is unstable ${ }^{102}$. Sister-chromatid cohesion is important for the co-orientation of sister kinetochores in both budding and fission yeast. In the absence of $R E C 8$, co-orientation of sister kinetochores during meiosis I is lost and sister chromatids segregate randomly ${ }^{30,56}$. Does this reflect a specific requirement for the meiotic cohesins in coorientation, or simply a need for sister chromatids to be held together by cohesion for them to co-orient?
This question has been addressed in budding and fission yeast by the replacement of Rec8 with Scc1 $(\operatorname{Mcd} 1) / \operatorname{Rad} 21$, but contrasting results were obtained. In budding yeast, $\mathrm{Scc} 1$ (Mcd1) can adequately support co-orientation, although it cannot support the maintenance of centromeric cohesion (see above, and REF. 72). In fission yeast, however, $\operatorname{Rec} 8$, but not $\operatorname{Rad} 21$, can support sister-kinetochore co-orientation ${ }^{73}$. Perhaps only Rec8-containing cohesin complexes can form cohesion at centromeres during meiosis in fission yeast.

\section{Meiosis l: factors required for co-orientation}

The proteins that are involved in promoting co-orientation during meiosis I were first identified, and are most well-characterized, in budding yeast. Mam1 (monopolar microtubule attachment during meiosis I), Csml (chromosome segregation in meiosis I) and Lrs4 (loss of rDNA silencing-4), which, together, form the 'MONOPOLIN' complex, are required for sister-kinetochore mono-orientation $^{72,103}$. In the absence of MAM1, CSM1 or LRS4, sister kinetochores attach to microtubules in a bipolar manner in meiosis I (REFS 72, 103). Consistent with its role in kinetochore co-orientation, Mam1 localizes to kinetochores during G2 and metaphase I, but dissociates from kinetochores at the onset of anaphase I (REF. 72). Unlike Mam1, Csm1 and Lrs4 are not meiosis specific as they are also present during the mitotic cell cycle, when they reside in the nucleolus ${ }^{103}$. During G2 and metaphase I of the meiotic cell cycle, Csm1 and Lrs4 leave the nucleolus and associate with kinetochores, together with Mam1. These results indicate that a monopolin complex forms at kinetochores during meiosis I and suppresses the biorientation of sister kinetochores.

An important insight into how the monopolin complex is regulated came from two studies on the budding yeast Polo-like kinase Cdc5 (REF. 75,76). Depletion of Cdc5 during meiosis revealed that, in addition to its role in the removal of cohesin ${ }^{76}$ and processing of recombination intermediates ${ }^{75}, C D C 5$ is required for the co-orientation of sister kinetochores, which is probably due to a failure to localize the monopolin complex to kinetochores ${ }^{75,76}$. $\mathrm{Cdc} 5$ is required for both the release of Lrs 4 from the nucleolus ${ }^{75}$ and the efficient phosphorylation of Mam1 (REF. 75,76), which suggests that phosphorylation by Cdc5 regulates the function of the monopolin complex.

A homologue of the monopolin component Csm1, Pcs1, has been identified in fission yeast ${ }^{103}$. In contrast to Csm1, Pcs1 is found at both kinetochores and in nucleoli during the mitotic cell cycle, and, surprisingly, Pcs 1 is required for accurate chromosome segregation during mitosis and meiosis II, but not meiosis I. A likely explanation for the defect in chromosome segregation in pcs1 mutants is the MEROTELIC attachment of a single chromatid $^{103}$ (FIG. 6). How can the opposite functions of Csm 1 and Pcs 1 be reconciled in terms of a common biochemical activity? An attractive hypothesis stems from the difference in the number of microtubules that attach to each kinetochore in the two yeasts ${ }^{103}$. In budding yeast, each sister chromatid binds just a single microtubule and so, merotelic attachment cannot occur $^{104}$. By contrast, fission yeast kinetochores (like the 

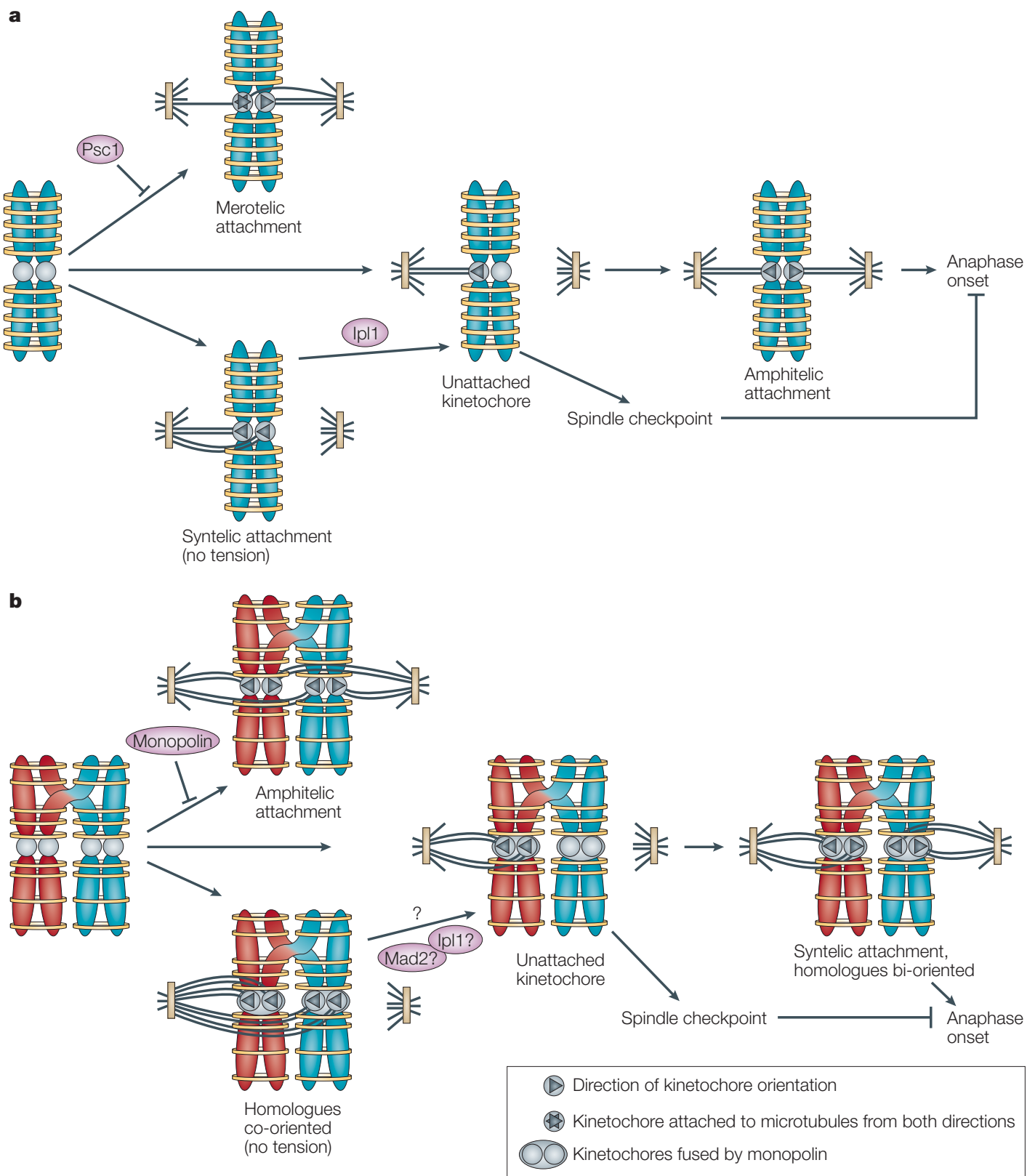

Figure 6 | Kinetochore orientation in mitosis and meiosis. a | In mitosis and meiosis II, fission yeast Pcs1 prevents the merotelic attachment of kinetochores to the microtubular spindle, perhaps by 'clamping' together adjacent microtubulebinding sites on the kinetochore. Merotelic attachment (that is, the attachment of a single kinetochore to microtubules from both poles) is avoided in budding yeast as there is only one microtubule-binding site per kinetochore ${ }^{104}$. Unattached kinetochores trigger the activation of the spindle checkpoint, which inhibits anaphase-promoting-complex/cyclosome-Cdc20 (APC/C ${ }^{C d 220}$ ), thereby preventing the degradation of securin and the loss of cohesin (yellow). Syntelic (or monopolar) attachments are destabilized by the Aurora-B kinase Ipl1, at least in budding yeast, which perhaps senses the lack of tension across sister kinetochores. Only when stable amphitelic (or bipolar) attachments are generated does anaphase onset occur. b | In meiosis I, sister kinetochores must be co-oriented and homologues bi-oriented. In budding yeast, the monopolin complex inhibits amphitelic attachment, perhaps by 'clamping' together microtubule-binding sites on adjacent sister kinetochores. The spindle checkpoint also functions in meiosis I and is presumably also activated in response to unattached kinetochores. There must also be a mechanism in meiosis to ensure that homologues are bi-oriented. Such a mechanism could correct homologue co-orientation by sensing the lack of tension across chiasmata and destabilize microtubule attachments on one homologue (see question mark). Note that in contrast to mitosis and meiosis II, the correct microtubule attachment at meiosis I would not generate tension across sister kinetochores. It is not known whether Ipl1 is capable of destabilizing syntelic attachments in meiosis I, but if it is, Ipl1 must somehow be prevented from doing so, perhaps by the monopolin complex. In budding yeast, the spindle-checkpoint component mitotic-arrest deficient (Mad)2 also seems to have a role in correcting kinetochore orientation during meiosis I, although another spindle-checkpoint component, Mad3, does not share this role (REFS 107,108). 
kinetochores of most other organisms) have several microtubule-binding sites ${ }^{105}$, and therefore require a mechanism to ensure that all binding sites on a kinetochore capture microtubules from the same pole to avoid merotelic attachment. It has been suggested ${ }^{103}$ that Pcs1 provides this activity by 'clamping' together microtubules from the same pole at the kinetochore. An analogous role for Csm1 during meiosis I can be envisioned. It is likely that both sister kinetochores bind a microtubule during meiosis I in budding yeast, and Csm 1 might function to 'clamp' these two microtubule-binding sites together.

If Pcsl is not required for meiosis I, which factors ensure the co-orientation of kinetochores in fission yeast meiosis I? Rec 8 and Bub1 are two such factors ${ }^{73,91}$, but other, as-yet-unknown proteins are likely to be involved because haploid cells that are engineered to undergo meiosis do not establish co-orientation, even though Rec8 is assembled onto the chromosomes ${ }^{102}$. Co-orientation does, however, occur in these cells if they receive mating pheromone. This indicates that mating-pheromone signalling, which is one of the events that usually precedes meiosis, triggers the expression of genes that are required for sister-kinetochore co-orientation in fission yeast.

\section{Meiosis I: allowing co-orientation}

During mitosis, anaphase onset does not occur until all pairs of sister chromatids have attached to the mitotic spindle in a bipolar manner; that is, they are bi-oriented (BOX 1; FIG. 6). Two factors contribute to establishing bipolar attachment. First, the spindle checkpoint senses the presence of unattached kinetochores and delays cellcycle progression until all kinetochores are attached (reviewed in REF. 106; see also BOX 1). The spindle checkpoint also functions during meiosis I, at least in yeast, and is likely to sense attachment defects ${ }^{107,108}$. The second factor that contributes to bipolar attachment in mitosis in budding yeast is the Aurora-B-like protein kinase Ipl1, which senses whether kinetochores are bi-oriented (perhaps by sensing tension at the kinetochore that is exerted by the pulling force of microtubules) ${ }^{109,110}$. If kinetochores are not bi-oriented, and therefore tension on kinetochores is absent, Ipl1 severs these faulty microtubule-kinetochore attachments. Unattached kinetochores are then sensed by the spindle checkpoint (BOX 1) and anaphase onset is inhibited.

In contrast to mitosis, when co-oriented kinetochores represent faulty attachments, co-oriented kinetochores are the rule during meiosis I (FIG.6). Sister kinetochores have to be co-oriented for homologues to segregate away from each other. How does this work? At least in budding yeast, the monopolin complex probably prevents kinetochores from bi-orienting, perhaps by fusing sister kinetochores by clamping together microtubule-binding sites on adjacent sister kinetochores. Linkages, such as chiasmata, between homologues could create tension at kinetochores. Bivalents attaching to microtubules that emanate from the same pole would be detected due to the absence of tension at kinetochores, and such attachments would be severed - perhaps by an Ipl1-mediated mechanism. Unattached kinetochores would then lead to activation of the spindle checkpoint and arrest in metaphase I. Elegant experiments in grasshopper spermatocytes support this idea ${ }^{111}$. However, achiasmatic mutants in budding yeast progress through both meiotic divisions despite the absence of tension on kinetochores ${ }^{112}$. Furthermore, cells that lack MAM1 orient kinetochores in an exclusively bipolar manner ${ }^{72}$ despite the presence of chiasmata, which should provide the necessary tension to allow for at least some bivalents to bi-orient on the meiosis-I spindle. These observations indicate that, in addition to tension-sensing mechanisms, other controls are involved in establishing proper kinetochore-microtubule attachments during meiosis I.

\section{The meiosis-I-meiosis-II transition}

In mitosis, DNA replication during $\mathrm{S}$ phase and chromosome segregation in $\mathrm{M}$ phase alternate to maintain the ploidy of daughter cells. This alternation of $S$ and $\mathrm{M}$ phases in mitosis is achieved through fluctuations in mitotic CDK activity (FIG. 2). A particular curiosity of the meiotic cell cycle is the lack of a DNA-replication phase between meiosis I and meiosis II. At the meiosis-I-meiosis-II transition, conditions must be established that both trigger meiotic spindle disassembly (low CDK activity) and prevent the formation of pre-replicative complexes (high CDK activity). How are these two apparently opposite conditions met by the cell-cycle machinery? Insights into this question have been obtained from the yeast and frog systems.

Work in frog oocytes has shown that an intermediate level of CDK activity is retained between meiosis I and meiosis II, and that this is necessary to prevent DNA replication ${ }^{113,114}$. Even though complete CDK inactivation interferes with the meiotic cell-cycle programme, the partial inactivation of CDKs that occurs during the meiosis-I-meiosis-II transition seems not to be necessary for this transition in frog oocytes. When APC/C $\mathrm{C}^{\mathrm{Cd} 20}$ activity is inhibited and CDK activity remains elevated between meiosis I and meiosis II, meiosis II occurs ${ }^{71}$. Although this does not exclude a role for the APC/C in the meiosis-I-meiosis-II transition (perhaps in cooperation with an accessory factor that is different from Cdc20), it does show that the levels of CDK activity do not need to be substantially lowered. However, completion of meiosis I in the frog is inhibited by injection of non-degradable cyclin B (REF. 115), which leaves open the possibility that a subtle shift in the balance between the rate of synthesis and degradation of cyclin B could regulate the meiosis-I-meiosis-II transition, as has been suggested for the mouse $\mathrm{e}^{116}$.

In budding yeast, lowering of the CDK activity during the meiosis-I-meiosis-II transition is important. Although some meiosis-II events can occur without downregulation of CDK activity between meiosis I and meiosis II, proper coordination of chromosome segregation is lost, with disastrous consequences ${ }^{117,118}$. In budding yeast, the protein phosphatase Cdc14 was found to be required, as it is in mitosis, for the downregulation of B-type cyclins and exit from meiosis I 


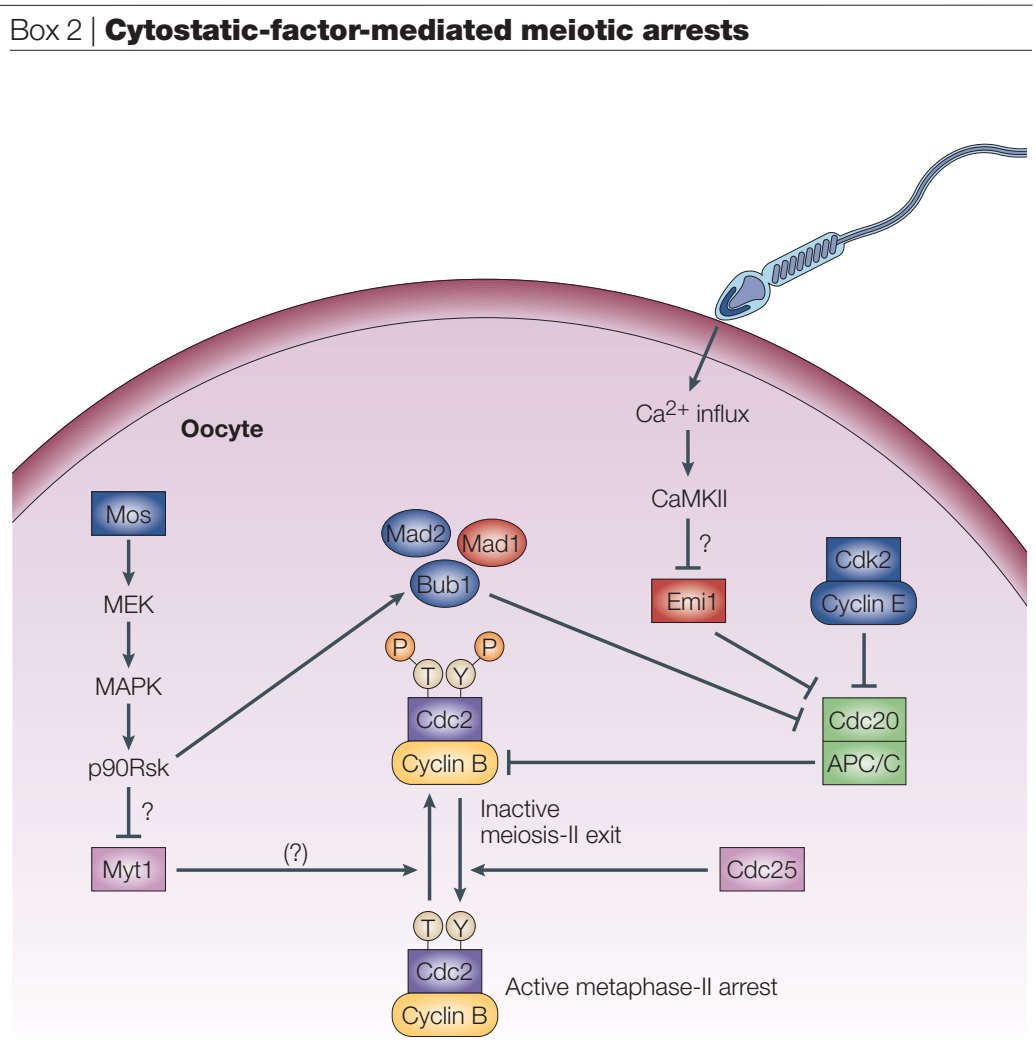

Masui and Markert first described an activity, known as cytostatic factor (CSF), that causes frog (Xenopus laevis) oocytes to arrest in metaphase II (REF. 147). CSF activity comprises at least three, possibly redundant, pathways that converge on the inhibition of the anaphase-promoting complex/cyclosome (APC/C), therefore preventing the degradation of cyclin $\mathrm{B}$. The Mos-mitogen-activated-protein-kinase (MAPK) pathway is one of these pathways ${ }^{120}$. Mos is present from G2 until after fertilization and is required for metaphase-II arrest in mouse oocytes ${ }^{148-150}$. Furthermore, Mos, MAPK and p90Rsk behave like CSF in their ability to induce cellcycle arrest when injected into mitotically dividing embryonic cells ${ }^{121,151-153}$. However, fruitfly (Drosophila melanogaster) Mos (DMOS) is not required for metaphase-I arrest of oocytes ${ }^{154}$. Activated p90Rsk phosphorylates and activates the $\mathrm{APC} / \mathrm{C}$ inhibitor and spindle-checkpoint component Bubl, which inhibits APC/C activity ${ }^{155}$. Two other spindle-checkpoint components, mitotic-arrest deficient (Mad)1 and Mad2, are also required for Mos-mediated APC/C inhibition ${ }^{156}$. Interestingly, CSF arrest occurs with all kinetochores attached to the spindle, a situation in which the spindle checkpoint is normally silenced, which indicates a more general role for spindle-checkpoint components in regulating APC/C activity. The contribution of Myt1 in mediating the downstream effects of the Mos-MAPK cascade is likely to be minor.

The second pathway that contributes to the inhibition of the APC/C involves cyclindependent kinase (Cdk)2-cyclin-E. Cyclin-E levels rise before meiosis II (REF. 157) and Cdk2-cyclin-E can inhibit the APC/C (REF. 155,158). The third pathway that mediates $\mathrm{APC} / \mathrm{C}$ inhibition in metaphase II is the APC/C inhibitor early meiotic induction (Emi) 1 (REF. 159). Emil directly binds to the APC/C activator, Cdc20 (also known as Fizzy), and inhibits it ${ }^{160}$. Depletion of Emil is sufficient to cause cyclin-B degradation and release from the metaphase-II arrest, which indicates that Emil is a key factor in maintaining a CSF arrest ${ }^{159}$.

Fertilization of the frog oocyte is associated with a rise in intracellular $\mathrm{Ca}^{2+}(\mathrm{REF} .161)$, which is sensed by the calmodulin-dependent kinase (CaMKII $)^{162}$. The targets of CaMKII in triggering release from CSF-mediated arrest are not known, although both Mad1 and Emil, but not Mos, need to be inactivated before release from the metaphase-II arrest can occur $^{70,159}$. Components shown in blue are involved in establishing CSF arrest, and those in red need to be inactivated before release from the arrest. MEK, MAPK and ERK (extracellular signal-regulated kinase) kinase. The figure is adapted from REF. 159.
(REFS 117,118). In the absence of Cdc14 or its regulator, the Cdc14 early anaphase release (FEAR) network, or in the presence of a non-degradable version of B-type cyclins, the meiosis-I spindle does not break down and meiosis-II spindles do not form ${ }^{117,118}$. These results indicate that CDK downregulation is critical for meiosis-I spindle disassembly. Surprisingly, however, other meiosis-II events occur in the absence of CDK downregulation, which leads to a meiosis-II-like segregation occurring on the meiosis-I spindle ${ }^{117,118}$. These observations indicate that, in budding yeast, downregulation of meiotic CDKs between meiosis I and meiosis II is important to ensure that chromosome segregation is coupled to the meiotic spindle cycle ${ }^{117,118}$.

How is residual CDK activity retained between the two meiotic divisions? Two mechanisms seem to contribute to this in frog oocytes: first, the partial inhibition of cyclin-B degradation upon exit from meiosis I; and second, the increased synthesis of cyclin B upon entry into meiosis II (REFS 71,119,120). Although the molecular details are not known, both processes seem to be mediated by the p90Rsk kinase, which functions downstream of the Mos-mitogen-activated-protein-kinase (MAPK) pathway ${ }^{71,113,121} \cdot$ p90Rsk is required for the partial inhibition of APC/C (REF. 120). The chromokinesin Xkid also seems to have a role in the meiosis-I-meiosis-II transition, which is independent of its function in metaphase chromosome alignment ${ }^{122}$. Furthermore, the retention of CDK activity between meiosis I and meiosis II depends on keeping two kinases, Wee1 and Myt1, inactive, as they phosphorylate and thereby inhibit Cdc2. Weel activity is inhibited because residual CDK activity is sufficient to overcome the low amount of Wee1 (REFS 114,123). Myt1 is kept inactive through phosphorylation by p90Rsk (REF. 124) and probably also by Plk1, which functions downstream of the Mos-MAPK pathway. In fission yeast, the $m e s 1^{+}$gene might be important for retaining CDK activity between the two meiotic divisions. In the absence of $m e s 1^{+}$, cells complete meiosis I but fail to enter meiosis II (REF. 125). This phenotype is similar to that exhibited by budding yeast cells that lack the B-type cyclins Clb1, Clb3 and Clb4 (REF. 126,127), which raises the possibility that mes $1^{+}$somehow functions to prevent complete CDK inactivation during the meiosis-I-meiosis-II transition.

\section{Meiosis II}

The specialized meiosis-I chromosome segregation is followed by a second chromosome-segregation phase, meiosis II. How CDKs are reactivated to allow entry into meiosis II is not known, although, at least in the frog, it depends on the synthesis of new B-type cyclins ${ }^{119}$. Meiosis-II chromosome segregation closely resembles mitosis. Sister kinetochores are bi-oriented, which is presumably due to the fact that the monopolin complex is no longer at kinetochores ${ }^{72,103}$. The centromeric cohesin complex, which escaped cleavage in meiosis I, resists the pulling force of the meiosis-II spindle before anaphase-II onset and so prevents the premature separation of sister chromatids (FIG. 4). At the onset of anaphase II, at least in budding yeast, separase once again becomes active and, as 
CSP

(cytostatic factor)

A cytoplasmic factor that is

responsible for the arrest of

oocytes at meiosis II there is no longer protection at the centromere, cleaves the remaining Rec8, which triggers the separation of sister chromatids to opposite poles.

Meiosis-II chromosome segregation, like the onset of meiosis I, is under developmental control. In most, if not all, vertebrates including frogs, mice and humans, oocytes arrest in metaphase II awaiting fertilization. The controls that bring about this cell-cycle arrest are summarized in BOX 2 (for recent reviews, see REFS 48,49).

\section{Concluding remarks}

During meiosis, parental chromosomes are shuffled and sorted into gametes for transmission to the next generation. The process of meiosis depends on the establishment of specialized interactions between chromosomes, coupled with modifications of the cellcycle machinery. Errors in these pathways result in defective chromosome segregation, which accounts for many birth defects and the majority of miscarriages in humans. It is worth noting that, in humans, up to $10 \%$ of all conceptions are estimated to be aneuploid ${ }^{128}$. Most chromosome-missegregation events in human meiosis occur as a result of improper segregation of homologues during meiosis I (REF. 128). An important goal for the future is to understand how the controls that regulate this unique chromosome-segregation event are put in place. Only then can we begin to understand what goes wrong.
1. Hubner, K. et al. Derivation of oocytes from mouse embryonic stem cells. Science 300, 1251-1256 (2003).

2. Toyooka, Y., Tsunekawa, N., Akasu, R. \& Noce, T. Embryonic stem cells can form germ cells in vitro. Proc. Natt Acad. Sci. USA 100, 11457-11462 (2003).

3. Geijsen, N. et al. Derivation of embryonic germ cells and male gametes from embryonic stem cells. Nature 427, 148-154 (2004).

4. Yamamoto, M. Regulation of meiosis in fission yeast. Cell Struct. Funct. 21, 431-436 (1996)

5. Honigberg, S. M. \& Purnapatre, K. Signal pathway integration in the switch from the mitotic cell cycle to meiosis in yeast. J. Cell Sci. 116, 2137-2147 (2003).

6. Chu, S. et al. The transcriptional program of sporulation in budding yeast. Science 282, 699-705 (1998).

7. Primig, M. et al. The core meiotic transcriptome in budding yeasts. Nature Genet. 26, 415-423 (2000).

8. Dirick, L., Goetsch, L., Ammerer, G. \& Byers, B. Regulation of meiotic S phase by Ime2 and a Clb5,6-associated kinase in Saccharomyces cerevisiae. Science 281, 1854-185 (1998).

Shows that Ime2 has a central role in promoting entry into pre-meiotic S phase. Ime2 mediates the destruction of the S-phase inhibitor, Sic1, and the activation of S-phase CDKs.

9. Bolte, M., Steigemann, P., Braus, G. H. \& Irniger, S. Inhibition of APC-mediated proteolysis by the meiosis-specific protein kinase Ime2. Proc. Natl Acad. Sci. USA 99, 4385-4390 (2002).

10. Watanabe, Y., Shinozaki-Yabana, S., Chikashige, Y., Hiraoka, Y. \& Yamamoto, M. Phosphorylation of RNA-binding protein controls cell cycle switch from mitotic to meiotic in fission yeast. Nature 386, 187-190 (1997).

11. Kitamura, K. et al. Phosphorylation of Mei2 and Ste11 by Pat1 kinase inhibits sexual differentiation via ubiquitin proteolysis and 14-3-3 protein in fission yeast. Dev. Cell 1 389-399 (2001).

12. Li, P. \& McLeod, M. Molecular mimicry in development: identification of ste $11^{+}$as a substrate and $m e i 3^{+}$as a pseudosubstrate inhibitor of ran 1+ kinase. Cell 87, 869-880 (1996).

13. Yamamoto, M., Imai, Y. \& Watanabe, Y. in The Molecular and Cellular Biology of the Yeast Saccharomyces (eds Pringle, J. R. Broach, J. R. \& Jones, E. W.) 1037-1106 (Cold Spring Harbor Laboratory Press, New York, 1997).

14. Watanabe, Y. \& Yamamoto, M. S. pombe mei2+ encodes an RNA-binding protein essential for premeiotic DNA synthesis and meiosis I, which cooperates with a novel RNA species meiRNA. Cell 78, 487-498 (1994).

15. Stuart, D. \& Wittenberg, C. CLB5 and CLB6 are required for premeiotic DNA replication and activation of the meiotic S/M checkpoint. Genes Dev. 12, 2698-2710 (1998).

16. Benjamin, K. R., Zhang, C., Shokat, K. M. \& Herskowitz, I. Control of landmark events in meiosis by the CDK Cdc28 and the meiosis-specific kinase Ime2. Genes Dev. 17. 1524-1539 (2003).

The authors show that the CDK Cdc28 is required for pre-meiotic $S$ phase. They also find that Ime 2 is required for the meiotic divisions, in addition to controlling entry into pre-meiotic $S$ phase.

17. Newlon, C. S. Yeast chromosome replication and segregation. Microbiol. Rev. 52, 568-601 (1988).

18. Collins, I. \& Newlon, C. S. Chromosomal DNA replication initiates at the same origins in meiosis and mitosis. Mol. Cell. Biol. 14, 3524-3534 (1994).

19. Simchen, G. Are mitotic functions required in meiosis? Genetics 76, 745-753 (1973).
20. Ofir, Y., Sagee, S., Guttmann-Raviv, N., Pnueli, L. \& Kassir, Y. The role and regulation of the preRC component $\mathrm{Cdc} 6$ in the initiation of premeiotic DNA replication. Mol. Biol. Cell 15, 2230-2242 (2004).

21. Cha, R. S. Weiner, B. M. Keeney, S., Dekker, J. \& Kleckner, N. Progression of meiotic DNA replication is modulated by interchromosomal interaction proteins, negatively by Spo $11 \mathrm{p}$ and positively by Rec8p. Genes Dev. 14, 493-503 (2000). Pre-meiotic DNA replication takes longer than premitotic DNA replication in all organisms tested. Evidence that preparation for recombination is one factor that affects the length of pre-meiotic $S$ phase was obtained through the observation that the absence of Rec8 or Spo11 alters the length of S phase.

22. Davis, L et al. The Saccharomyces cerevisiae MUM2 gene interacts with DNA replication maching and is equed interacts with the DNA replication machinery and is required for melotic levels of doublestrand ber 1179-1189 (2001)

23. Borde, V., Goldman, A. S. \& Lichten, M. Direct coupling between meiotic DNA replication and recombination initiation. Science 290, 806-809 (2000).

When replication is delayed on a specific proportion of the chromosome, DSB formation at the same location is delayed by a similar amount, which shows that DNA replication and the appearance of DSBs are directly coupled.

24. Smith, K. N. Penkner, A., Ohta, K., Klein, F. \& Nicolas, A. B-type cyclins CLB5 and CLB6 control the initiation of recombination and synaptonemal complex formation in yeast meiosis. Curr. Biol. 11, 88-97 (2001).

25. Bergerat, A. et al. An atypical topoisomerase Il from Archaea with implications for meiotic recombination. Nature $\mathbf{3 8 6}$, 414-417 (1997)

26. Keeney, S., Giroux, C. N. \& Kleckner, N. Meiosis-specific DNA double-strand breaks are catalyzed by Spo11, a member of a widely conserved protein family. Cell $\mathbf{8 8}$ 375-384 (1997)

27. Cervantes, M. D., Farah, J. A. \& Smith, G. R. Meiotic DNA breaks associated with recombination in S. pombe. Mol. Cell 5, 883-888 (2000).

28. Murakami, H. \& Nurse, P. Regulation of premeiotic S phase and recombination-related double-strand DNA breaks during meiosis in fission yeast. Nature Genet. 28, 290-293 (2001).

29. Molnar, M., Bahler, J., Sipiczki, M. \& Kohli, J. The rec8 gene of $S$ chizosaccharomyces pombe is involved in linear element formation, chromosome pairing and sister-chromatid cohesion during meiosis. Genetics 141, 61-73 (1995).

30. Klein, F. et al. A central role for cohesins in sister chromatid cohesion, formation of axial elements, and recombination during yeast meiosis. Cell 98, 91-103 (1999). Smc3 and the meiosis-specific Scc1(Mcd1)/Rad21 homologue Rec8 are required for cohesion between sister chromatids in budding yeast. Rec8 is lost from chromosome arms during meiosis I and from centromeric regions during meiosis II.

31. Watanabe, Y., Yokobayashi, S., Yamamoto, M. \& Nurse, P. Pre-meiotic $S$ phase is linked to reductional chromosome segregation and recombination. Nature 409, 359-363 (2001).

32. Uhlmann, F. \& Nasmyth, K. Cohesion between sister chromatids must be established during DNA replication. Curr. Biol. 8, 1095-1101 (1998).

33. Paques, F. \& Haber, J. E. Multiple pathways of recombination induced by double-strand breaks in Saccharomyces cerevisiae. Microbiol. Mol. Biol. Rev. 63, 349-404 (1999).

34. Zickler, D. \& Kleckner, N. Meiotic chromosomes: integrating structure and function. Annu. Rev. Genet. 33, 603-754 (1999).
35. Bishop, D. K. \& Zickler, D. Early decision: meiotic crossove interference prior to stable strand exchange and synapsis. Cell 117, 9-15 (2004).

36. Allers, T. \& Lichten, M. Differential timing and control of noncrossover and crossover recombination during meiosis. Cell 106, 47-57 (2001).

Previous models of recombination proposed that $\mathrm{COs}$ and NCOs are generated by differential resolution of DHJs. This paper shows that NCO heteroduplex products are formed at the same time as DHJs. These results indicate that $\mathrm{CO}$ are formed by the resolution of DHJs, whereas NCOs are generated by a different pathway.

37. Borner, G. V., Kleckner, N. \& Hunter, N. Crossover/noncrossover differentiation, synaptonemal complex formation, and regulatory surveillance at the leptotene/zygotene transition of meiosis. Cell 117, 29-45 (2004).

38. Hunter, N. \& Kleckner, N. The single-end invasion: an asymmetric intermediate at the double-strand break to double-Holliday junction transition of meiotic recombination. Cell 106, 59-70 (2001)

39. Fung, J. C., Rockmill, B., Odell, M. \& Roeder, G. S. Imposition of crossover interference through the nonrandom distribution of synapsis initiation complexes. Cell 116, 795-802 (2004).

40. Roeder, G. S. \& Bailis, J. M. The pachytene checkpoint. Trends Genet. 16, 395-403 (2000).

41. Murakami, H. \& Nurse, P. DNA replication and damage checkpoints and meiotic cell cycle controls in the fission and budding yeasts. Biochem. J. 349, 1-12 (2000).

42. Yoshida, K. et al. The mouse RecA-like gene $D m c 1$ is required for homologous chromosome synapsis during meiosis. Mol. Cell 1, 707-718 (1998).

43. Pittman, D. L. et al. Meiotic prophase arrest with failure of chromosome synapsis in mice deficient for Dmc1, a germline-specific RecA homolog. Mol. Cell 1, 697-705 (1998)

44. Leu, J. Y. \& Roeder, G. S. The pachytene checkpoint in S. cerevisiae depends on Swe1-mediated phosphorylation of the cyclin-dependent kinase Cdc28. Mol. Cell 4, 805-814 (1999).

45. Pak, J. \& Segall, J. Role of Ndt80, Sum1, and Swe1 as targets of the meiotic recombination checkpoint that control exit from pachytene and spore formation in Saccharomyces cerevisiae. Mol. Cell. Biol. 22, 6430-6440 (2002).

46. Tung, K. S., Hong, E. J.\& Roeder, G. S. The pachytene checkpoint prevents accumulation and phosphorylation of the meiosis-specific transcription factor Ndt80. Proc. Natl Acad. Sci. USA 97, 12187-12192 (2000).

47. Lindgren, A. et al. The pachytene checkpoint in Saccharomyces cerevisiae requires the Sum1 transcriptional repressor. EMBO J. 19, 6489-6497 (2000).

48. Kishimoto, T. Cell-cycle control during meiotic maturation. Curr. Opin. Cell Biol. 15, 654-663 (2003).

49. Tunquist, B. J. \& Maller, J. L. Under arrest: cytostatic factor (CSF)-mediated metaphase arrest in vertebrate eggs. Genes Dev. 17, 683-710 (2003)

50. Dernburg, A. F. et al. Meiotic recombination in C. elegans initiates by a conserved mechanism and is dispensable for homologous chromosome synapsis. Cel/ 94, 387-398 (1998).

51. Steiner, W. W., Schreckhise, R. W. \& Smith, G. R. Meiotic DNA breaks at the $S$. pombe recombination hot spot M26. Mol. Cell 9, 847-855 (2002)

52. Baudat, F., Manova, K., Yuen, J. P., Jasin, M. \& Keeney, S. Chromosome synapsis defects and sexually dimorphic meiotic progression in mice lacking Spo11. Mol. Cell 6 , 989-998 (2000). 
53. Romanienko, P. J. \& Camerini-Otero, R. D. The mouse Spo11 gene is required for meiotic chromosome synapsis. Mol. Cell 6, 975-987 (2000).

54. Gruber, S., Haering, C. H. \& Nasmyth, K. Chromosoma cohesin forms a ring. Cell 112, 765-777 (2003). The authors carried out a series of experiments, the results of which are consistent with a model in which cohesin forms a ring around sister chromatids.

55. Pasierbek, P. et al. A Caenorhabditis elegans cohesion protein with functions in meiotic chromosome pairing and disjunction. Genes Dev. 15, 1349-1360 (2001).

56. Watanabe, Y. \& Nurse, P. Cohesin Rec8 is required for reductional chromosome segregation at meiosis. Nature 400, 461-464 (1999)

57. Lee, J., Iwai, T., Yokota, T. \& Yamashita, M. Temporally and spatially selective loss of Rec8 protein from meiotic chromosomes during mammalian meiosis. J. Cell Sci. 116, 2781-2790 (2003)

58. Eijpe, M., Offenberg, H., Jessberger, R., Revenkova, E. \& Heyting, C. Meiotic cohesin REC8 marks the axial elements of rat synaptonemal complexes before cohesins SMC1 $\beta$ and SMC3. J. Cell Biol. 160, 657-670 (2003).

59. Kitajima, T. S., Yokobayashi, S., Yamamoto, M. \& Watanabe, Y. Distinct cohesin complexes organize meiotic chromosome domains. Science 300, 1152-1155 (2003). In fission yeast, the Scc3 homologue Psc3 forms part of the cohesin complex. In meiosis, Psc3 is part of the cohesin complex at centromeric regions; however, a meiosis-specific variant, Rec11, substitutes for Psc3 pericentromere and inner centromere have different requirements for their assembly.

60. DeVeaux, L. C. \& Smith, G. R. Region-specific activators of meiotic recombination in Schizosaccharomyces pombe. Genes Dev. 8, 203-210 (1994).

61. Krawchuk, M. D., DeVeaux, L. C. \& Wahls, W. P. Meiotic chromosome dynamics dependent upon the rec $8^{+}$, rec $10^{*}$ and rec $11^{+}$genes of the fission yeast Schizosaccharomyces pombe. Genetics 153, 57-68 (1999).

62. Sumara, l. et al. The dissociation of cohesin from chromosomes in prophase is regulated by Polo-like kinase. Mol. Cell 9, 515-525 (2002).

\section{In vertebrates, the bulk of cohesin is removed from} chromosome arms during prophase and prometaphase in a mechanism that requires the Polo-like kinase.

63. Losada, A., Hirano, M. \& Hirano, T. Cohesin release is required for sister chromatid resolution, but not for condensin-mediated compaction, at the onset of mitosis. Genes Dev. 16, 3004-3016 (2002).

Removal of cohesin from chromosome arms during prophase and pro-metaphase in the frog requires both Polo-like kinase and Aurora B.

64. Buonomo, S. B. et al. Disjunction of homologous chromosomes in meiosis I depends on proteolytic cleavage of the meiotic cohesin Rec8 by separin. Cell 103, 387-398 (2000).

\section{Cleavage of Rec 8 by separase is required for the} resolution of chiasmata and the disjunction of homologues to opposite poles in budding yeast.

65. Kitajima, T. S., Miyazaki, Y., Yamamoto, M. \& Watanabe, Y. Rec8 cleavage by separase is required for meiotic nuclear divisions in fission yeast. EMBO J. 22, 5643-5653 (2003).

66. Davis, E. S. et al. Multiple subunits of the Caenorhabditis elegans anaphase-promoting complex are required for chromosome segregation during meiosis I. Genetics 160 805-813 (2002).

67. Siomos, M. F. et al. Separase is required for chromosome segregation during meiosis I in Caenorhabditis elegans. Curr. Biol. 11, 1825-1835 (2001).

68. Terret, M. E. et al. The meiosis I-to-meiosis II transition in mouse oocytes requires separase activity. Curr. Biol. 13 1797-1802 (2003).

69. Herbert, M. et al. Homologue disjunction in mouse oocytes requires proteolysis of securin and cyclin B1. Nature Cell Biol. 5, 1023-1025 (2003).

References 67-69 show that the requirement for separase in the disjunction of homologues at meiosis I is conserved.

70. Peter, M. et al. The APC is dispensable for first meiotic anaphase in Xenopus oocytes. Nature Cell Biol. 3, 83-87 (2001).

71. Taieb, F. E., Gross, S. D., Lewellyn, A. L. \& Maller, J. L Activation of the anaphase-promoting complex and degradation of cyclin $\mathrm{B}$ is not required for progression from meiosis I to II in Xenopus oocytes. Curr. Biol. 11, 508-513 (2001).

\section{References 70 and 71 show that the APC is not} required for meiosis $I$ in the frog.

72. Toth, A. et al. Functional genomics identifies monopolin: a kinetochore protein required for segregation of homologs during meiosis I. Cell 103, 1155-1168 (2000).

Reports the identification of the budding yeast protein Mam1 - the first protein known to mediate monopolar attachment of kinetochores during meiosis $\mathrm{I}$.

73. Yokobayashi, S., Yamamoto, M. \& Watanabe, Y. Cohesins determine the attachment manner of kinetochores to spind microtubules at meiosis I in fission yeast. Mol. Cell. Biol. 23, 3965-3973 (2003).

74. Alexandru, G., Uhlmann, F., Mechtler, K., Poupart, M. A. \& Nasmyth, K. Phosphorylation of the cohesin subunit Scc1 by Polo/Cdc5 kinase regulates sister chromatid separation in yeast. Cell 105, 459-472 (2001).

75. Clyne, R. K. et al. Polo-like kinase Cdc5 promotes chiasmata formation and cosegregation of sister centromeres at meiosis I. Nature Cell Biol. 5, 480-485 (2003).

76. Lee, B. H. \& Amon, A. Role of Polo-like kinase CDC5 in programming meiosis I chromosome segregation. Science 300, 482-486 (2003).

References 75 and 76 show that the Polo-like kinase Cdc5 has several roles in meiosis-I chromosome segregation.

77. Grether, M. E. \& Herskowitz, I. Genetic and biochemical characterization of the yeast spo12 protein. Mol. Biol. Cell 10, 3689-3703 (1999).

78. Lee, B. H., Amon, A. \& Prinz, S. Spo13 regulates cohesin cleavage. Genes Dev. 16, 1672-1681 (2002).

79. Shonn, M. A., McCarroll, R. \& Murray, A. W. Spo13 protects meiotic cohesin at centromeres in meiosis I. Genes Dev. 16, 1659-1671 (2002)

80. Kerrebrock, A. W. Moore, D. P., Wu, J. S. \& Orr-Weaver, T. L. Mei-S332, a Drosophila protein required for sister-chromatid cohesion, can localize to meiotic centromere regions. Cell $\mathbf{8 3}$, 247-256 (1995)

\section{$\mathrm{MEI}-\mathrm{S} 332$ localizes to centromeric regions from} during meiosis until centromeric cohesion is lost at anaphase II.

81. Moore, D. P., Page, A. W., Tang, T. T., Kerrebrock, A. W. \& Orr-Weaver, T. L. The cohesion protein MEl-S332 localizes to condensed meiotic and mitotic centromeres until sister chromatids separate. J. Cell Biol. 140, 1003-1012 (1998).

82. Kerrebrock, A W Miyazaki, W. Y. Birnby, D. \& Orr-Weaver, T. L. The Drosophila mei-S332 gene promotes sister-chromatid cohesion in meiosis following kinetochore differentiation. Genetics 130, 827-841 (1992).

83. Katis, V. L., Galova, M., Rabitsch, K. P., Gregan, J. \& Nasmyth, K. Maintenance of cohesin at centromeres after meiosis I in budding yeast requires a kinetochore-associated protein related to MEI-S332. Curr Biol. 14, 560-572 (2004).

84. Kitajima, T. S., Kawashima, S. A. \& Watanabe, Y. The conserved kinetochore protein shugoshin protects centromeric cohesion during meiosis. Nature 427, 510-517 (2004).

85. Marston, A. L., Tham, W. H., Shah, H. \& Amon, A. A genome-wide screen identifies genes required for centromeric cohesion. Science. 303, 1367-1370 (2004).

86. Rabitsch, K. P. et al. Two fission yeast homologs of Drosophila Mei-S332 are required for chromosome segregation during meiosis I and II. Curr. Biol. 14, 287-301 (2004).

\section{References 83-86 describe the identification of} homologues of Mei-S332, known as shugoshins. Fission and budding yeast $\mathrm{Sgo} 1$ are required to retain Rec8 at centromeres until meiosis II.

87. LeBlanc, H. N., Tang, T. T., Wu, J. S. \& Orr-Weaver, T. L. The mitotic centromeric protein MEI-S332 and its role in sisterchromatid cohesion. Chromosoma 108, 401-411 (1999).

88. Blat, Y. \& Kleckner, N. Cohesins bind to preferential sites along yeast chromosome III, with differential regulation along arms versus the centric region. Cell 98, 249-259 (1999).

89. Megee, P. C., Mistrot, C., Guacci, V. \& Koshland, D. The centromeric sister chromatid cohesion site directs Mcd1p binding to adjacent sequences. Mol. Cell 4, 445-450 (1999).

O. Tanaka, T., Cosma, M. P., Wirth, K. \& Nasmyth, K. Identification of cohesin association sites at centromeres and along chromosome arms. Cel/ 98, 847-858 (1999).

91. Bernard, P., Maure, J. F. \& Javerzat, J. P. Fission yeast Bub1 is essential in setting up the meiotic pattern of chromosome segregation. Nature Cell Biol. 3, 522-526 (2001).

92. Nonaka, N. et al. Recruitment of cohesin to heterochromatic regions by Swi6/HP1 in fission yeast. Nature Cell Biol. $\mathbf{4}$, 89-93 (2002)

93. Bernard, P. et al. Requirement of heterochromatin for cohesion at centromeres. Science 294, 2539-2542 (2001)

94. Lopez, J. M., Karpen, G. H. \& Orr-Weaver, T. L. Sisterchromatid cohesion via MEI-S332 and kinetochore assembly are separable functions of the Drosophila centromere. Curr. Biol. 10, 997-1000 (2000).

95. Blower, M. D. \& Karpen, G. H. The role of Drosophila CID in kinetochore formation, cell-cycle progression and heterochromatin interactions. Nature Cell Biol. 3, 730-739 (2001).
96. Weber S. A et al. The kinetochore is an enhancer of pericentric cohesin binding. PLOS Biol. 2, E260 (2004).

97. Nasmyth, K. Disseminating the genome: joining, resolving, and separating sister chromatids during mitosis and meiosis. Annu. Rev. Genet. 35, 673-745 (2001).

98. Goldstein, L. S. Kinetochore structure and its role in chromosome orientation during the first meiotic division in male D. melanogaster. Cell 25, 591-602 (1981).

99. Suja, J. A., de la Torre, J., Gimenez-Abian, J. F., Garcia de la Vega, C. \& Rufas, J. S. Meiotic chromosome structure. Kinetochores and chromatid cores in standard and B chromosomes of Arcyptera fusca (Orthoptera) revealed by silver staining. Genome 34, 19-27 (1991).

100. Paliulis, L. V. \& Nicklas, R. B. The reduction of chromosome number in meiosis is determined by properties built into the chromosomes. J. Cell Biol. 150, 1223-1232 (2000). Meiotic chromosome behaviour was shown to be a chromosome intrinsic property in grasshopper spermatocytes. Transplantion of a meiosis-1 chromosome to a meiosis-II chrosion the

01. Davis, L. \& Smith, G. R. Nonrandom homolog segregation at meiosis I in Schizosaccharomyces pombe mutants lacking recombination. Genetics 163, 857-874 (2003).

102. Yamamoto, A. \& Hiraoka, Y. Monopolar spindle attachment of sister chromatids is ensured by two distinct mechanisms at the first meiotic division in fission yeast. EMBO J. 22 , 2284-2296 (2003)

103. Rabitsch, K. P. et al. Kinetochore recruitment of two nucleolar proteins is required for homolog segregation in meiosis I. Dev. Cell 4, 535-548 (2003).

104. Winey, M. et al. Three-dimensional ultrastructural analysis of the Saccharomyces cerevisiae mitotic spindle. J. Cell Biol. 129, 1601-1615 (1995).

105. Ding, R., McDonald, K. L. \& McIntosh, J. R. Threedimensional reconstruction and analysis of mitotic spindles from the yeast, Schizosaccharomyces pombe. J. Cell Biol. 120, 141-151 (1993).

106. Gardner, R. D. \& Burke, D. J. The spindle checkpoint: two transitions, two pathways. Trends Cell Biol. 10, 154-158 (2000)

107. Shonn, M. A., McCarroll, R. \& Murray, A. W. Requirement of the spindle checkpoint for proper chromosome segregation in budding yeast meiosis. Science 289, 300-303 (2000).

108. Shonn, M. A., Murray, A. L. \& Murray, A. W. Spindle checkpoint component Mad2 contributes to biorientation of homologous chromosomes. Curr. Biol. 13, 1979-1984 (2003).

109. Dewar, H., Tanaka, K., Nasmyth, K. \& Tanaka, T. U. Tension between two kinetochores suffices for their bi-orientation on the mitotic spindle. Nature 428, 93-97 (2004).

110. Biggins, S. \& Murray, A. W. The budding yeast protein kinase Ipl1/Aurora allows the absence of tension to activate the spindle checkpoint. Genes Dev. 15, 3118-3129 (2001).

111. Li, X. \& Nicklas, R. B. Mitotic forces control a cell-cycle checkpoint. Nature 373, 630-632 (1995).

112. Klapholz, S., Waddell, C. S. \& Esposito, R. E. The role of the SPO11 gene in meiotic recombination in yeast. Genetics 110, 187-216 (1985).

113. Furuno, N. et al. Suppression of DNA replication via Mos function during meiotic divisions in Xenopus oocytes. EMBO J. 13, 2399-2410 (1994).

Mos and the retention of a modest level of CDK activity between meiosis I and meiosis II is required to prevent DNA replication between the meiotic divisions.

114. Iwabuchi, M., Ohsumi, K., Yamamoto, T. M., Sawada, W. \& Kishimoto, T. Residual Cdc2 activity remaining at meiosis I exit is essential for meiotic M-M transition in Xenopus oocyte extracts. EMBO J. 19, 4513-4523 (2000).

115. Huchon, D., Rime, H., Jessus, C. \& Ozon, R. Control of metaphase I formation in Xenopus oocyte: effects of an indestructible cyclin B and of protein synthesis. Biol. Cell 77 , 133-141 (1993).

116. Ledan, E., Polanski, Z., Terret, M. E. \& Maro, B. Meiotic maturation of the mouse oocyte requires an equilibrium between cyclin B synthesis and degradation. Dev. Biol. 232, 400-413 (2001).

117. Buonomo, S. B. et al. Division of the nucleolus and its release of $\mathrm{CDC1} 14$ during anaphase of meiosis I depends on separase, SPO12, and SLK19. Dev. Cell 4, 727-739 (2003).

118. Marston, A. L., Lee, B. H. \& Amon, A. The Cdc14 phosphatase and the FEAR network control meiotic spindle disassembly and chromosome segregation. Dev. Cell. 4 , 711-726 (2003).

References 117 and 118 show that downregulation of CDK activity by the FEAR network and Cdc14 is required for exit from meiosis I and for coupling of the meiotic chromosome-segregation cycle with development.

119. Hochegger, H. et al. New B-type cyclin synthesis is required between meiosis I and II during Xenopus oocyte maturation. Development 128, 3795-3807 (2001). 
120. Gross, S. D. et al. The critical role of the MAP kinase pathway in meiosis II in Xenopus oocytes is mediated by p90 ${ }^{\text {Fsk }}$. Curr. Biol. 10, 430-438 (2000).

121. Gross, S. D., Schwab, M. S., Lewellyn, A. L. \& Maller, J. L. Induction of metaphase arrest in cleaving Xenopus embryos by the protein kinase p90 Rsk. Science 286, 1365-1367 (1999). Mos-MAPK cascade.

122. Perez, L. H., Antonio, C., Flament, S., Vernos, I. \& Nebreda, A. R. Xkid chromokinesin is required for the meiosis I to meiosis || transition in Xenopus laevis oocytes. Nature Cell Biol. 4, 737-742 (2002)

123. Nakajo, N. et al. Absence of Wee1 ensures the meiotic cell cycle in Xenopus oocytes. Genes Dev. 14, 328-338 (2000).

124. Palmer, A., Gavin, A. C. \& Nebreda, A. R. A link between MAP kinase and $\mathrm{p} 34^{\mathrm{cod} 2} / \mathrm{cyclin} \mathrm{B}$ during oocyte maturation $90^{\text {rsk }}$ phosphorylates and inactivates the p34 $4^{\text {odc2 }}$ inhibitory kinase Myt1. EMBO J. 17, 5037-5047 (1998). p90Rsk phosphorylates and inhibits Myt1, thereby maintaining cyclinB-Cdc2 in the active form.

125. Shimoda, C., Hirata, A., Kishida, M., Hashida, T. \& Tanaka, K. Characterization of meiosis-deficient mutants by electron microscopy and mapping of four essential genes in the fission yeast Schizosaccharomyces pombe Mol Gen. Genet. 200, 252-257 (1985).

126. Grandin, N. \& Reed, S. I. Differential function and expression of Saccharomyces cerevisiae B-type cyclins in mitosis and meiosis. Mol. Cell. Biol. 13, 2113-2125 (1993).

127. Dahmann, C., Diffley, J. F. \& Nasmyth, K. A. S-phasepromoting cyclin-dependent kinases prevent re-replication by inhibiting the transition of replication origins to a prereplicative state. Curr. Biol. 5, 1257-1269 (1995).

128. Hassold, T. \& Hunt, P. To err (meiotically) is human: the genesis of human aneuploidy. Nature Rev. Genet. 2 280-291 (2001).

129. Guacci, V., Koshland, D. \& Strunnikov, A. A direct link between sister chromatid cohesion and chromosome condensation revealed through the analysis of MCD1 in S. cerevisiae. Cell 91, 47-57 (1997).

130. Michaelis, C., Ciosk, R. \& Nasmyth, K. Cohesins: chromosomal proteins that prevent premature separation of sister chromatids. Cell 91, 35-45 (1997).

131. Losada, A., Hirano, M. \& Hirano, T. Identification of Xenopus SMC protein complexes required for sister chromatid cohesion. Genes Dev. 12, 1986-1997 (1998).

32. Sumara, I., Vorlaufer, E., Gieffers, C., Peters, B. H. 8 Peters, J. M. Characterization of vertebrate cohesin complexes and their regulation in prophase. J. Cell Biol. 151 749-762 (2000).

133. Toth, A. et al. Yeast cohesin complex requires a conserved protein, Eco1 $\mathrm{p}^{\mathrm{Ctr}}$, to establish cohesion between siste chromatids during DNA replication. Genes Dev. 13, 320-333 (1999)

134. Tomonaga, T. et al. Characterization of fission yeast cohesin: essential anaphase proteolysis of Rad21 phosphorylated in the S phase. Genes Dev. 14, 2757-2770 (2000).

135. van Heemst, D., James, F., Poggeler, S., Berteaux-Lecellier, V. \& Zickler, D. Spo76p is a conserved chromosome morphogenesis protein that links the mitotic and meiotic programs. Cell 98, 261-271 (1999).

136. Hartman, T., Stead, K., Koshland, D. \& Guacci, V. Pds5p is an essential chromosomal protein required for both sister chromatid cohesion and condensation in Saccharomyces cerevisiae. J. Cell Biol. 151, 613-626 (2000).

137. Panizza, S., Tanaka, T., Hochwagen, A., Eisenhaber, F. \& Nasmyth, K. Pds 5 cooperates with cohesin in maintaining sister chromatid cohesion. Curr. Biol. 10, 1557-1564 (2000).

138. Tanaka, K., Hao, Z., Kai, M. \& Okayama, H. Establishment and maintenance of sister chromatid cohesion in fission yeast by a unique mechanism. EMBO J. 20, 5779-5790 (2001).

139. Wang, F., Yoder, J., Antoshechkin, I. \& Han, M Caenorhabditis elegans EVL-14/PDS-5 and SCC-3 are essential for sister chromatid cohesion in meiosis and mitosis. Mol. Cell. Biol. 23, 7698-7707 (2003).

140. Uhlmann, F., Lottspeich, F. \& Nasmyth, K. Sister-chromatid separation at anaphase onset is promoted by cleavage of the cohesin subunit Scc1. Nature 400, 37-42 (1999).

141. Uhlmann, F., Wernic, D., Poupart, M. A., Koonin, E. V. \& Nasmyth, K. Cleavage of cohesin by the $C D$ clan protease separin triggers anaphase in yeast. Cell 103, 375-386 (2000).
142. Waizenegger, I. C., Hauf, S., Meinke, A. \& Peters, J. M. Two distinct pathways remove mammalian cohesin from chromosome arms in prophase and from centrom anaphase. Cell 103, 399-410 (2000).

A separase-independent pathway removes cohesin from chromosome arms during prophase, whereas a separase-dependent pathway cleaves centromeric cohesin at the metaphase-anaphase transition.

143. Cohen-Fix, O., Peters, J. M., Kirschner, M. W. \& Koshland, D. Anaphase initiation in Saccharomyces cerevisiae is controlled by the APC-dependent degradation of the anaphase inhibitor Pds1p. Genes Dev. 10, 3081-3093 (1996)

144. Funabiki, H. et al. Cut2 proteolysis required for sisterchromatid seperation in fission yeast. Nature 381, 438-441 (1996).

145. Ciosk, R. et al. An ESP1-PDS1 complex regulates loss of sister chromatid cohesion at the metaphase to anaphase transition in yeast. Cell 93, 1067-1076 (1998).

146. Lew, D. J. \& Burke, D. J. The spindle assembly and spindle position checkpoints. Annu. Rev. Genet. 37, 251-282 2003)

147. Masui, Y. \& Markert, C. L. Cytoplasmic control of nuclear behavior during meiotic maturation of frog oocytes. J. Exp. Zool. 177, 129-145 (1971).

148. Hashimoto, N. et al. Parthenogenetic activation of oocytes in c-mos-deficient mice. Nature 370, 68-71 (1994).

149. Colledge, W. H., Carlton, M. B., Udy, G. B. \& Evans, M. J. Disruption of c-mos causes parthenogenetic development of unfertilized mouse eggs. Nature 370, 65-68 (1994).

150. Sagata, N., Watanabe, N., Vande Woude, G. F. \& lkawa, Y. The c-mos proto-oncogene product is a cytostatic factor responsible for meiotic arrest in vertebrate eggs. Nature $\mathbf{3 4 2}$. $512-518$ (1989)

151. Sagata, N., Daar, I., Oskarsson, M., Showalter, S. D. \& Vande Woude, G. F. The product of the mos proto-oncogene as a candidate 'initiator' for oocyte maturation. Science $\mathbf{2 4 5}$ 643-646 (1989)

152. Haccard, O. et al. Induction of metaphase arrest in cleaving Xenopus embryos by MAP kinase. Science $\mathbf{2 6 2}, \mathbf{1 2 6 2 - 1 2 6 5}$ (1993).

153. Bhatt, R. R. \& Ferrell, J. E., Jr. The protein kinase p90 rsk as an essential mediator of cytostatic factor activity. Science 286, 1362-1365 (1999).

154. Ivanovska, I., Lee, E., Kwan, K. M., Fenger, D. D. \& Orr-Weaver, T. L. The Drosophila MOS ortholog is not essential for meiosis. Curr. Biol. 14, 75-80 (2004).

155. Schwab, M. S. et al. Bub1 is activated by the protein kinase p90Rsk during Xenopus oocyte maturation. Curr. Biol. 11 $141-150$ (2001).

156. Tunquist, B. J., Eyers, P. A., Chen, L. G., Lewellyn, A. L. \& Maller, J. L. Spindle checkpoint proteins Mad1 and Mad2 are required for cytostatic factor-mediated metaphase arrest. J. Cell Biol. 163, 1231-1242 (2003).

157. Rempel, R. E., Sleight, S. B. \& Maller, J. L. Maternal Xenopus Cdk2-cyclin E complexes function during meiotic and early embryonic cell cycles that lack a G1 phase. J. Biol. Chem 270, 6843-6855 (1995)

158. D'Angiolella, V. et al. Role for cyclin-dependent kinase 2 in mitosis exit. Curr. Biol. 11, 1221-1226 (2001).

159. Reimann, J. D. \& Jackson, P. K. Emi1 is required for cytostatic factor arrest in vertebrate eggs. Nature $\mathbf{4 1 6}$ 850-854 (2002).

\section{The APC ${ }^{\mathrm{Cdc} 20}$ inhibitor Emi1 is both necessary and} sufficient for CSF arrest in frog oocytes.

160. Reimann, J. D. et al. Emi1 is a mitotic regulator that interacts with $\mathrm{Cdc} 2 \mathrm{O}$ and inhibits the anaphase promoting complex. Cell 105, 645-655 (2001).

161. Kubota, H. Y., Yoshimoto, Y., Yoneda, M. \& Hiramoto, Y. Free calcium wave upon activation in Xenopus eggs. Dev. Biol. 119, 129-136 (1987)

162. Lorca, T. et al. Calmodulin-dependent protein kinase II mediates inactivation of MPF and CSF upon fertilization of Xenopus eggs. Nature 366, 270-273 (1993)

163. Koepp, D. M., Harper, J. W. \& Elledge, S. J. How the cyclin became a cyclin: regulated proteolysis in the cell cycle. Cell 97, 431-434 (1999)

164. Morgan, D. O. Cyclin-dependent kinases: engines, clocks, and microprocessors. Annu. Rev. Cell Dev. Biol. 13, 261-291 (1997).

165. Harper, J. W., Burton, J. L. \& Solomon, M. J. The anaphase promoting complex: it's not just for mitosis any more. Genes Dev. 16, 2179-2206 (2002).
166. Mito, Y., Sugimoto, A. \& Yamamoto, M. Distinct developmental function of two Caenorhabditis elegans homologs of the cohesin subunit Scc1/Rad21. Mol. Biol. Cell 14, 2399-2409 (2003).

167. Chan, R. C. et al. Chromosome cohesion is regulated by a clock gene paralogue TIM-1. Nature 424, 1002-1009 (2003).

168. Warren, W. D. et al. The Drosophila RAD21 cohesin persists at the centromere region in mitosis. Curr. Biol. 10, 1463-1466 (2000).

169. Vass, S. et al. Depletion of Drad21/Scc1 in Drosophila cells eads to instability of the cohesin complex and disruption of mitotic progression. Curr. Biol. 13, 208-218 (2003).

170. Losada A Yokochi, T., Kobayashi, R. \& Hirano, T Identification and characterization of SAVScc3p subunits in the Xenopus and human cohesin complexes. J. Cell Biol. 150, 405-416 (2000).

171. Darwiche, N., Freeman, L. A. \& Strunnikov, A. Characterization of the components of the putative mammalian sister chromatid cohesion complex. Gene $\mathbf{2 3 3}$ 39-47 (1999).

172. Schmiesing, J. A. et al. Identification of two distinct human SMC protein complexes involved in mitotic chromosome dynamics. Proc. Natl Acad. Sci. USA 95, 12906-12911 (1998).

173. Cai, X., Dong, F., Edelmann, R. E. \& Makaroff, C. A. The Arabidopsis SYN1 cohesin protein is required for sister chromatid arm cohesion and homologous chromosome pairing. J. Cell Sci. 116, 2999-3007 (2003).

174. Prieto, I. et al. STAG2 and Rad21 mammalian mitotic cohesins are implicated in meiosis. EMBO Rep. 3, 543-550 (2002).

175. Parisi, S. et al. Rec8p, a meiotic recombination and sister chromatid cohesion phosphoprotein of the Rad21p family conserved from fission yeast to humans. Mol. Cell. Biol. 19, 3515-3528 (1999).

176. Parra, M. T. et al. Involvement of the cohesin Rad21 and SCP3 in monopolar attachment of sister kinetochores during mouse meiosis I. J. Cell Sci. 117, 1221-1234 (2004).

177. Xu, H. et al. A new role for the mitotic RAD21/SCC1 cohesin in meiotic chromosome cohesion and segregation in the mouse. EMBO Rep. 5, 378-384 (2004).

178. Pasierbek, P. et al. The Caenorhabditis elegans SCC-3 homologue is required for meiotic synapsis and for proper chromosome disjunction in mitosis and meiosis. Exp. Cell Res. 289, 245-255 (2003).

179. Prieto, I. et al. Mammalian STAG3 is a cohesin specific to sister chromatid arms in meiosis I. Nature Cell Biol. $\mathbf{3}$, 761-766 (2001).

180. Prieto, I. et al. Cohesin component dynamics during meiotic prophase I in mammalian oocytes. Chromosome Res. 12 197-213 (2004).

181. Revenkova, E., Eijpe, M., Heyting, C., Gross, B. \& Jessberger, R. Novel meiosis-specific isoform of mammalian smc1. Mol. Cell. Biol. 21, 6984-6998 (2001).

\section{Acknowledgements}

We are grateful to T. Orr-Weaver and members of the Amon laboratory for their critical reading of this manuscript. We apologize to our colleagues whose work was not discussed in this review due to space limitations.

Competing interests statement

The authors declare no competing financial interests.

\section{(2) Online links}

\section{DATABASES}

The following terms in this article are linked online to:

Flybase: $\mathrm{http}$ :/flybase bio indiana edu/

MEI-S332

Saccharomyces genome database:

http://www.yeastgenome.org/

Cdc5 | Cdc14 | Cdc28 | Ch14 | CLB1 | CLB3 | CLB4 | Clb5 | Clb6 | Csm1 | Emi1 | Esp1 | IME1 | IME2 | Iml3 | Ipl1 | Mam1 | MUM2 | Ndt80 | Pds5 |Rec8 | Scc1 | Scc3 | Sgo1 | Sic1 | Smc1 | Smc3 | Spo11 | Spo13 | Sum1 | Swe1

S. pombe gene database:

http://www.genedb.org/genedb/pombe/index.jsp Bub1 | Clr4 | Cnp1 | Mei2 | Mei3 | meiRNA | mes 1+ | Pat1 | Pcs1 |

Psc3 |Rad21 | Rec11 |Rec12 | Sgo2 | Swi6

Access to this links box is available online. 
E R R A T U M

\section{ERRATUM}

\section{MEIOSIS: CELL-CYCLE CONTROLS SHUFFLE AND DEAL}

\section{Adèle L. Marston \& Angelika Amon}

Nature Reviews Molecular Cell Biology 5, 983-997 (2004); doi:10.1038/nrm1526

On page 984 of this article, in Figure $1 \mathrm{~b}$, the homologous chromosomes were incorrectly shaded at the transition between meiosis I and meiosis II. A corrected version of the figure is shown below. The online versions of this article have been corrected.

b Sister chromatids

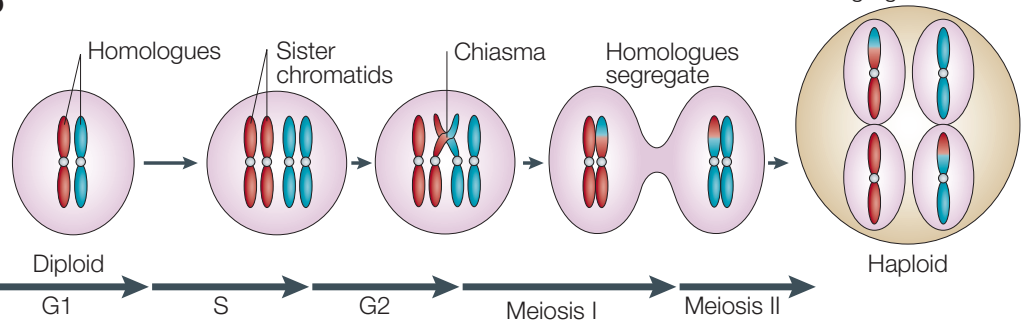

